\title{
A Review of Major Application Areas of Differential Evolution
}

\author{
V.P. Plagianakos ${ }^{1}$, D.K. Tasoulis ${ }^{2}$, and M.N. Vrahatis ${ }^{1}$ \\ 1 Computational Intelligence Laboratory, Department of Mathematics, \\ University of Patras Artificial Intelligence Research Center-UPAIRC, \\ University of Patras, GR-26110 Patras, Greece \\ \{vpp, vrahatis\}@math. upatras.gr \\ 2 Institute for Mathematical Sciences, Imperial College London, \\ South Kensington, London SW7 2PG, United Kingdom \\ d.tasoulis@imperial.ac.uk
}

Summary. In this chapter we present an overview of the major applications areas of differential evolution. In particular we pronounce the strengths of DE algorithms in tackling many difficult problems from diverse scientific areas, including single and multiobjective function optimization, neural network training, clustering, and real life DNA microarray classification. To improve the speed and performance of the algorithm we employ distributed computing architectures and demonstrate how parallel, multi-population DE architectures can be utilised in single and multiobjective optimization. Using data mining we present a methodology that allows the simultaneous discovery of multiple local and global minimizers of an objective function. At a next step we present applications of DE in real life problems including the training of integer weight neural networks and the selection of genes of DNA microarrays in order to boost predictive accuracy of classification models. The chapter concludes with a discussion on promising future extensions of the algorithm, and presents novel mutation operators, that are the result of a genetic programming procedure, as very interesting future research direction.

\section{Introduction}

Evolutionary Algorithms (EAs) refer to problem solving optimization algorithms which employ computational models of evolutionary processes. A variety of evolutionary algorithms have been proposed. The major ones include: Genetic Algorithms [21, 24], Evolutionary Programming [17, 19], Evolution Strategies [39], Genetic Programming [29], Particle Swarm Optimization [27], and the Differential Evolution algorithm [46]. All these algorithms share the common conceptual base of simulating the evolution of a population of individuals using a predefined set of operators. Commonly two kinds of operators are used: the selection and the search operators. The most widely used search operators are mutation and recombination.

The selection operator enforces the natural selection and the survival of the fittest on the population of individuals. The recombination and the mutation operators stochastically perturb the individuals providing efficient exploration of the search space. This 
perturbation is primarily controlled by the user defined recombination and mutation rates. Although simplistic from a biologist's point of view, these algorithms are sufficiently complex to provide robust and powerful search mechanisms and have shown their strength in solving hard optimization problems.

In this chapter we focus on the Differential Evolution algorithm and present some of its applications, including single and multiobjective function optimization, neural network training, clustering, and real life DNA microarray problems. Furthermore, we discuss promising future extensions of the algorithm by the incorporation of genetically programmed mutation operators.

\section{The Differential Evolution Algorithm}

More than ten years ago, Storn and Price [46] have presented a novel optimization method, called Differential Evolution (DE), which has been designed to handle nondifferentiable, nonlinear and multimodal objective functions. To fulfill this requirement, DE has been designed as a stochastic parallel direct search method, which utilizes concepts borrowed from the broad class of evolutionary algorithms, but requires few easily chosen control parameters. Early experimental results have shown that DE has good convergence properties and outperforms other well known evolutionary algorithms [45, 46]. In the following paragraphs we outline the workings of DE.

\subsection{The Workings of DE}

In each population, new individuals (vectors) are generated by the combination of randomly chosen vectors. This operation in our context can be referred as mutation. The outcoming vectors are then mixed with another predetermined vector - the target vector - and this operation can be called recombination. This operation yields the so-called trial vector. The trial vector is accepted for the next generation if and only if it reduces the value of the objective function $f$. This operation can be referred as selection. A high-level description of the above mentioned operators (for one generation) is given below:

Step 1. Do for each Vector

Step 2. MutantVector := MUTATION(Vector)

Step 3. TrialVector := RECOMBINATION(MutantVector)

Step 4. If $\mathrm{f}$ (TrialVector) $\leqslant \mathrm{f}$ (Vector)

Step 5. Vector := TrialVector

Step 6. EndIf

Step 7. EndDo

We now briefly review the two basic DE variation operators. The first DE operator we consider is mutation. Specifically, for each individual $x_{g}^{i}, i=1,2, \ldots, N P$, where $g$ denotes the current generation and $N P$ the number of individuals in the population, 
a new individual $v_{g+1}^{i}$ (mutant vector) is generated according to one of the following equations:

$$
\begin{aligned}
& v_{g+1}^{i}=x_{g}^{\text {best }}+\mu\left(x_{g}^{r_{1}}-x_{g}^{r_{2}}\right), \\
& v_{g+1}^{i}=x_{g}^{r_{1}}+\mu\left(x_{g}^{r_{2}}-x_{g}^{r_{3}}\right), \\
& v_{g+1}^{i}=x_{g}^{i}+\mu\left(x_{g}^{\text {best }}-x_{g}^{i}\right)+\mu\left(x_{g}^{r_{1}}-x_{g}^{r_{2}}\right), \\
& v_{g+1}^{i}=x_{g}^{\text {best }}+\mu\left(x_{g}^{r_{1}}-x_{g}^{r_{2}}\right)+\mu\left(x_{g}^{r_{3}}-x_{g}^{r_{4}}\right), \\
& v_{g+1}^{i}=x_{g}^{r_{1}}+\mu\left(x_{g}^{r_{2}}-x_{g}^{r_{3}}\right)+\mu\left(x_{g}^{r_{4}}-x_{g}^{r_{5}}\right),
\end{aligned}
$$

where $x_{g}^{\text {best }}$ is the best member of the previous generation; $\mu>0$ is a real parameter, called mutation constant, which controls the amplification of the difference between two individuals so as to avoid the stagnation of the search process; and $r_{1}, r_{2}, r_{3}, r_{4}, r_{5} \in\{1,2, \ldots, i-1, i+1, \ldots, N P\}$, are random integers mutually different and not equal to the running index $i$.

Trying to rationalize the above equations, we observe that Equation (2) is similar to the crossover operator used by some Genetic Algorithms and Equation (1) derives from it, when the best member of the previous generation is employed. Equations (3), (4) and (5) are modifications obtained by the combination of Equations (1) and (2). It is clear that more such relations can be generated using the above ones as building blocks. For example, the recently proposed trigonometric mutation operator [14] performs mutations with probability $\tau_{\mu}$ according to the following equation:

$$
\begin{aligned}
v_{g+1}^{i}= & \left(x_{g}^{r_{1}}+x_{g}^{r_{2}}+x_{g}^{r_{3}}\right) / 3+\left(p_{2}-p_{1}\right)\left(x_{g}^{r_{1}}-x_{g}^{r_{2}}\right)+ \\
& +\left(p_{3}-p_{2}\right)\left(x_{g}^{r_{2}}-x_{g}^{r_{3}}\right)+\left(p_{1}-p_{3}\right)\left(x_{g}^{r_{3}}-x_{g}^{r_{1}}\right),
\end{aligned}
$$

and with probability $\left(1-\tau_{\mu}\right)$ performs mutations according to Equation (2), where $\tau_{\mu}$ is a user defined parameter. The values of $p_{m}, m=\{1,2,3\}$ and $p^{\prime}$ are obtained through the following equations:

$$
\begin{aligned}
p_{1} & =\left|f\left(x_{g}^{r_{1}}\right)\right| / p^{\prime}, p_{2}=\left|f\left(x_{g}^{r_{2}}\right)\right| / p^{\prime}, p_{3}=\left|f\left(x_{g}^{r_{3}}\right)\right| / p^{\prime}, \text { and } \\
p^{\prime} & =\left|f\left(x_{g}^{r_{1}}\right)\right|+\left|f\left(x_{g}^{r_{2}}\right)\right|+\left|f\left(x_{g}^{r_{3}}\right)\right| .
\end{aligned}
$$

For the rest of the chapter, we call $\mathrm{DE}_{1}$ the differential evolution algorithm that uses Equation (1) as the mutation operator, $\mathrm{DE}_{2}$ the algorithm that uses Equation (2), and so on.

The recombination operator is subsequently applied to further increase the diversity of the mutant individuals. To this end, the resulting individuals are combined with other predetermined individuals, called the target individuals. Specifically, for each component $l(l=1,2, \ldots, n)$ of the mutant individual $v_{g+1}^{i}$, we randomly choose a real number $r$ in the interval $[0,1]$. Then, we compare this number with the recombination constant, $\rho$. If $r \leqslant \rho$, then we select, as the $l$-th component of the trial individual $u_{g+1}^{i}$, the $l$-th component of the mutant individual $v_{g+1}^{i}$. Otherwise, the $l$-th component of the target vector $x_{g+1}^{i}$ becomes the $l$-th component of the trial vector. This operation yields the trial individual. 
Finally, the trial vector $u_{g+1}^{i}$ is accepted for the next generation only if it reduces the value of the objective function. Thus:

$$
x_{g+1}^{i}= \begin{cases}u_{g+1}^{i}, & \text { if } f\left(u_{g+1}^{i}\right)<f\left(x_{g}^{i}\right), \\ x_{g}^{i}, & \text { otherwise. }\end{cases}
$$

To prevent an individual from surviving indefinitely, we can employ the concept of aging [45]. To this end, each vector is randomly assigned a maximum age, i.e. an integer from the interval $[\alpha, \beta]$, where $\alpha$ and $\beta$ are the minimum and the maximum possible age, respectively. At each iteration, the age of each vector is increased by one, and if it exceeds its maximum age then the individual "dies". This individual is then replaced by another vector randomly chosen from the current population. Note that it is desirable the best individual of the population not to be eliminated.

\subsection{Implementation of Parallel Evolutionary Algorithms}

Parallel processing, that is the method of having many small tasks solve one large problem, has emerged as a key enabling technology in modern computing. As a result of the demand for higher performance, lower cost, and sustained productivity, the past several years have witnessed an ever-increasing acceptance and adoption of parallel processing, both for high-performance scientific computing and for more general-purpose applications. Exploiting recent software advances [1, 20], collections of heterogeneous computers can be used as a coherent and flexible concurrent computational resource. These technologies have allowed the vast number of individual personal computers available in most scientific laboratories to be used as parallel machines at no, or at a very low, cost. Network interfaces, linking individual computers, are necessary to produce such pools of computational power.

EAs, as well as DEs, are easily parallelized [32, 43]. There are two typical models for EA parallelization. The first uses fine grained parallelism, so each individual is represented by a processor. This creates certain problems when the number of processors available is limited or when the individual's fitness to reproduce needs to be evaluated over the whole population. The second model, maps an entire subpopulation to a processor. Thus each subpopulation evolves independently toward a solution. This allows each subpopulation to develop its own solution uniquely. Then, the best individual of each subpopulation is propagated to other subpopulations, according to the selected network topology. This operation is called migration. This model is called the Parallel Evolutionary Algorithm (PEA).

Usually, the topology of the PEA is a ring, i.e. the best individuals from each subpopulation are allowed to migrate to the next subpopulation of the ring. This concept reduces the migration between the subpopulations and consequently the messages between the processors. The migration of the best individuals is controlled by the migration constant, $\varphi \in(0,1)$. At each iteration, a random number from the interval $(0,1)$ is uniformly chosen and compared with the migration constant. If the migration constant is bigger, then the best individuals of each subpopulation migrate and take the place of a randomly selected individual (different from the best one) in the next subpopulation; otherwise no migration is permitted. We have experimentally found that a migration 
constant, $\varphi \simeq 0.5$, is a good choice, since it allows each subpopulation to evolve for some iterations before the migration phase actually occur.

\section{Function Optimization Using DE}

Let us know consider the minimization problem of finding global minima of a continuous nonlinear, (possibly) nondifferentiable, multimodal objective function $f$. More specifically, our goal is to locate global minimizers $x_{t}^{*}$ of the real-valued objective function $f: \mathcal{E} \rightarrow \mathbb{R}:$

$$
f\left(x_{t}^{*}\right) \leqslant f(x), \quad \forall x \in \mathcal{E},
$$

where $t=1,2, \ldots$, and the compact set $\mathcal{E} \subseteq \mathbb{R}^{n}$ is a $n$-dimensional scaled translation of the unit hypercube.

\subsection{Single Objective Optimization}

Firstly, we will evaluate the performance of the DE algorithms employing seven single objective test functions. We briefly describe them below.

Test Function 1: Sphere

$$
f_{1}(x)=\sum_{j=1}^{5} x_{j}^{2}, \quad x_{j} \in[-5.12,5.12] .
$$

The sphere test function is a considered to be a simple minimization problem. The minimum is $f_{1}(0,0, \ldots, 0)=0$.

\section{Test Function 2: Rosenbrock's Saddle}

$$
f_{2}(x)=100 \cdot\left(x_{1}^{2}-x_{2}\right)^{2}+\left(1-x_{1}\right)^{2}, \quad x_{j} \in[-2.048,2.048] .
$$

This is a two-dimensional test function, which is known to be relatively difficult to minimize. The minimum is $f_{2}(1,1)=0$.

\section{Test Function 3: Step Function}

$$
f_{3}(x)=30+\sum_{j=1}^{5}\left\lfloor x_{j}\right\rfloor, \quad x_{j} \in[-5.12,5.12],
$$

where the floor function $\lfloor x\rfloor$ gives the largest integer less than or equal to $x$. The minimum of this function is $f_{3}(-5-\xi, \ldots,-5-\xi)=0$, where $\xi \in[0,0.12]$. This function exhibits many flat regions that can cause search stagnation.

Test Function 4: Quartic Function

$$
f_{4}(x)=\sum_{j=1}^{30}\left(j \cdot x_{j}^{4}+\eta\right), \quad x_{j} \in[-1.28,1.28] .
$$

This is test function is designed to evaluate the behavior of minimization algorithms in the presence of noise. To this end, $\eta$ is a random variable following the uniform distribution in the range $[0,1]$. The inclusion of $\eta$ makes $f_{4}$ more difficult to optimize. The functional minimum of the function is $f_{4}(0,0, \ldots, 0) \leqslant 30 \cdot E[\eta]=15$, where $E[\eta]$ is the expectation of $\eta$. 
Test Function 5: Shekel's Foxholes

$$
f_{5}(x)=\frac{1}{0.002+\psi_{1}(x)}, \quad x_{j} \in[-65.536,65.536],
$$

where, $\psi_{1}(x)=\sum_{i=0}^{24} \frac{1}{1+i+\sum_{j=1}^{2}\left(x_{j}-a_{i j}\right)^{6}}$. The parameters for this function are:

$$
\begin{aligned}
& a_{i 1}=\{-32,-16,0,16,32\}, \text { where } i=\{0,1,2,3,4\} \text { and } a_{i 1}=a_{i \bmod 5,1} \\
& a_{i 2}=\{-32,-16,0,16,32\}, \text { where } i=\{0,5,10,15,20\} \text { and } a_{i 2}=a_{i+k, 2},
\end{aligned}
$$

and $k=\{1,2,3,4\}$. The global minimum of $f_{5}(-32,-32)=0.998004$.

\section{Test Function 6: Corana Parabola}

$$
f_{6}(x)=\sum_{j=1}^{4}\left\{\begin{array}{l}
\psi_{2}\left(x_{j}\right), \text { if }\left|x_{j}-z_{j}\right|<0.05 \\
\psi_{3}\left(x_{j}\right), \text { otherwise. }
\end{array}\right.
$$

where $\psi_{2}\left(x_{j}\right)=0.15\left(z_{j}-0.05 \operatorname{sign}\left(z_{j}\right)\right)^{2} d_{j}, \psi_{3}\left(x_{j}\right)=d_{j} x_{j}^{2}, z_{j}=\left\lfloor 5\left|x_{j}\right|+\right.$ $0.49999\rfloor \operatorname{sign}\left(x_{j}\right) 0.2$ and $d_{j}=\{1,1000,10,100\}$. The Corana test function defines a paraboloid with axes parallel to the coordinate axes. The function is characterized by a multitude of local minima, increasing in depth as one moves closer to the origin. The global minimum of the function is $f_{6}(x)=0$, for $x_{j} \in(-0.05,0.05)$.

\section{Test Function 7: Griewangk's Function}

$$
f_{7}(x)=\sum_{j=1}^{10} \frac{x_{j}^{2}}{4000}-\prod_{j=1}^{10} \cos \left(\frac{x_{j}}{\sqrt{j}}\right)+1, \text { where } x_{j} \in[-400,400] .
$$

This test function is riddled with local minima. The global minimum of the function is $f_{7}(0,0, \ldots, 0)=0$.

\section{Experimental Results on Parallel Differential Evolution}

Here we present results of the Parallel Differential Evolution (PARDE) algorithm [47, on the above test functions. In Table 1 the parameter setup used in the numerical experiments conducted is summarized. Specifically, $D$ denotes the dimensionality of the problem, NP stands for the size of the subpopulation assigned to each of the processors employed, $g$ is the maximum number of generations allowed, finally, $\mu$ and $\rho$ are the values of the mutation and recombination constants, respectively.

Little effort has been devoted to the selection of the values of $N P, \mu$ and $\rho$ since the scope of these experiments is to study extensively, the implications of information sharing in a parallel environment, which is controlled by the migration constant, $\varphi$. It is worth noting that further performance improvements can be achieved by further fine-tuning $N P, \mu$, and $\rho$. The parameter $\tau_{\mu}$ used by the trigonometric mutation strategy, Equation (6), was set to 0.1. Figure 1 illustrates the speedup achieved by assigning each subpopulation to a different processor, relative to assigning all subpopulations to 
Table 1. Parameter values

\begin{tabular}{lrrrrr}
\hline \multicolumn{1}{c}{ Test function } & $D$ & $N P$ & $g$ & $\mu$ & $\rho$ \\
\hline Sphere function & 5 & 30 & 1000 & 0.9 & 0.3 \\
Rosenbrock's saddle & 2 & 30 & 1000 & 0.9 & 0.5 \\
Step function & 5 & 20 & 1000 & 0.8 & 0.3 \\
Quartic function & 30 & 100 & 2000 & 0.8 & 0.5 \\
Shekel's foxholes & 2 & 30 & 1000 & 0.9 & 0.3 \\
Corana's parabola & 4 & 15 & 2000 & 0.4 & 0.2 \\
Griewangk's function & 10 & 50 & 10000 & 1.0 & 0.3 \\
\hline
\end{tabular}

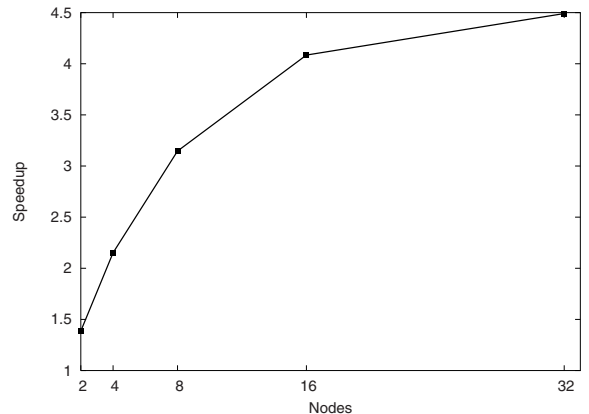

Fig. 1. Speedup using up to 32 nodes

a single processor. To obtain the plotted values, the algorithm performed 1000 generations with a migration constant equal to 0.5 . Table 2 reports the mean number of generations required to locate the global minimum of each test function, averaged over all the considered mutation constants. It is clear from Table 2 that the best performing mutation strategy, for all test problems, was the first one. Furthermore, Griewangk's function appears to be the hardest to minimize. Figures 24 illustrate the performance of the 16-node model for all the considered mutation strategies, on all the test functions, for a particular migration constant. In all the $3 D$ plots, the mutation strategies are given by the $x$-axis, the test functions by the $y$-axis, and finally, the mean number of generations required is reported in the $z$-axis. Concerning the overall performance of the alternative mutation strategies, the worst performance is exhibited by strategies 5 and 6 Strategies 1 and 3 appear to be the most efficient and robust.
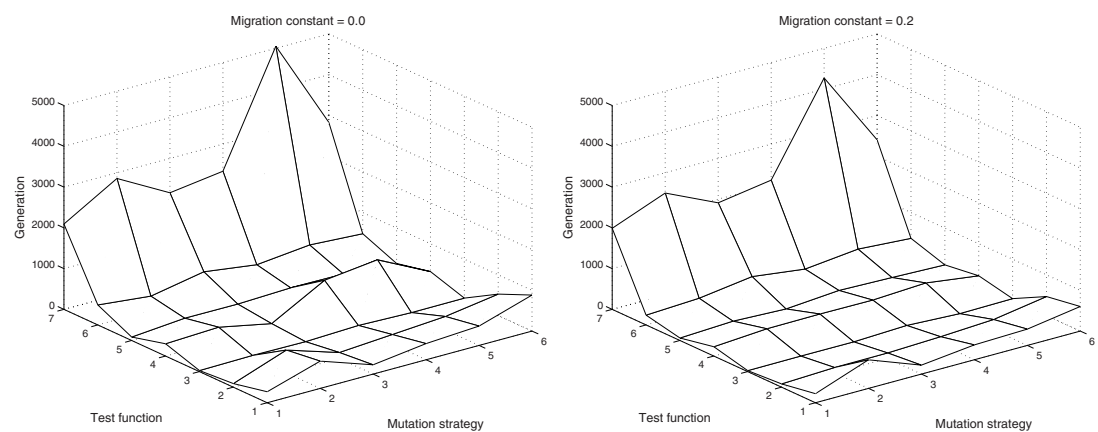

Fig. 2. Parallel DE results 
Table 2. Mean number of generations for the 16-node model

\begin{tabular}{lrrrrrr}
\hline Test function & \multicolumn{7}{c}{ Mutation Strategy } \\
& 1 & 2 & 3 & 4 & 5 & 6 \\
\hline Sphere & $\mathbf{2 3 1 . 0 9}$ & 611.58 & 247.02 & 341.08 & 409.79 & 642.02 \\
Rosenbrock & $\mathbf{8 2 . 2 1}$ & 386.01 & 130.14 & 220.77 & 271.39 & 371.59 \\
Step & $\mathbf{2 1 . 9 1}$ & 76.85 & 25.48 & 47.59 & 25.40 & 31.59 \\
Quartic & $\mathbf{2 4 4 . 9 2}$ & 249.90 & 260.21 & 406.13 & 454.18 & 244.11 \\
Shekel & $\mathbf{6 3 . 3 9}$ & 136.96 & 96.21 & 186.91 & 158.30 & 101.55 \\
Corana & $\mathbf{2 8 2 . 3 5}$ & 364.09 & 513.36 & 329.48 & 491.01 & 398.85 \\
Griewangk & $\mathbf{1 8 7 2 . 1 7}$ & 1885.19 & 1975.61 & 2644.47 & 4448.66 & 2434.45 \\
\hline
\end{tabular}
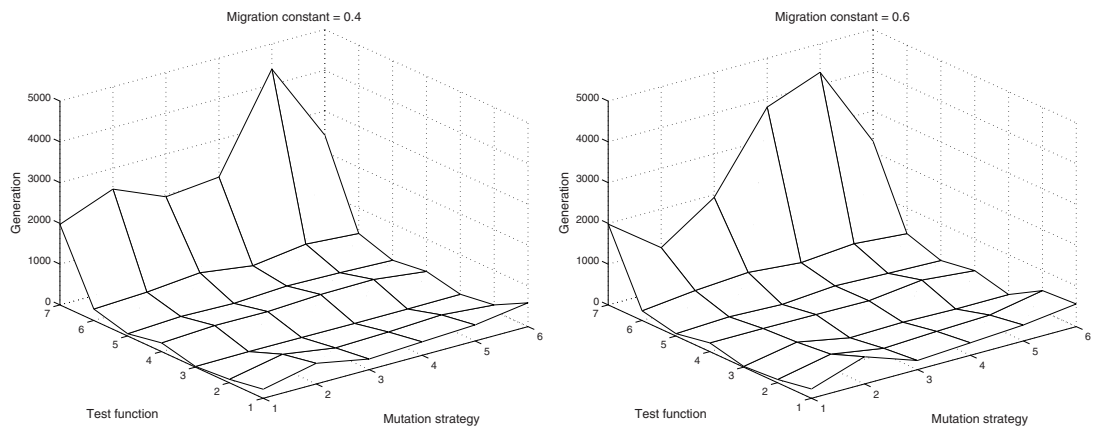

Fig. 3. Parallel DE results
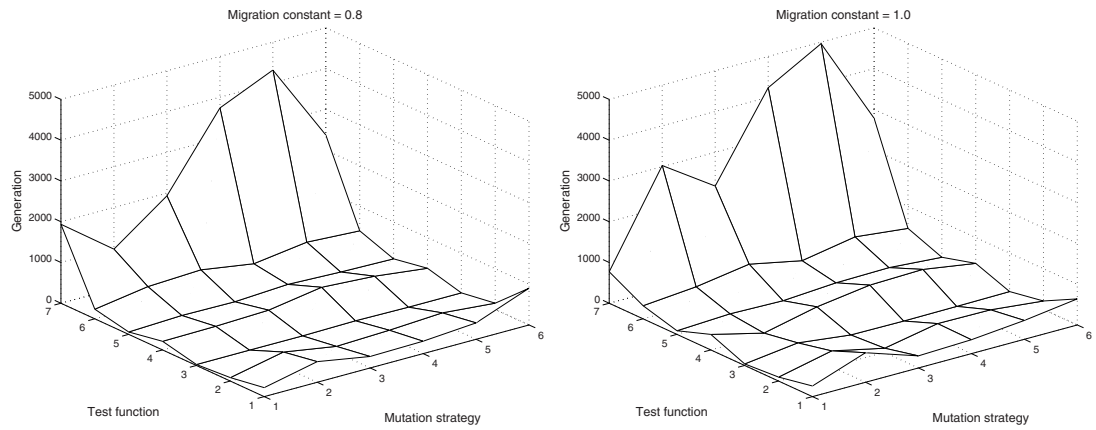

Fig. 4. Parallel DE results

Figure 5 exhibits the mean number of generations required for each migration constant, $\varphi \in[0,1]$ with stepsize 0.1 , for all the mutation strategies on the Griewangk test function. It is evident that selecting the appropriate migration constant has a significant impact on the performance of the algorithm. Moreover, it appears that setting $\varphi$ close to one or to zero can lead to a substantial increase in the number of generations required. 


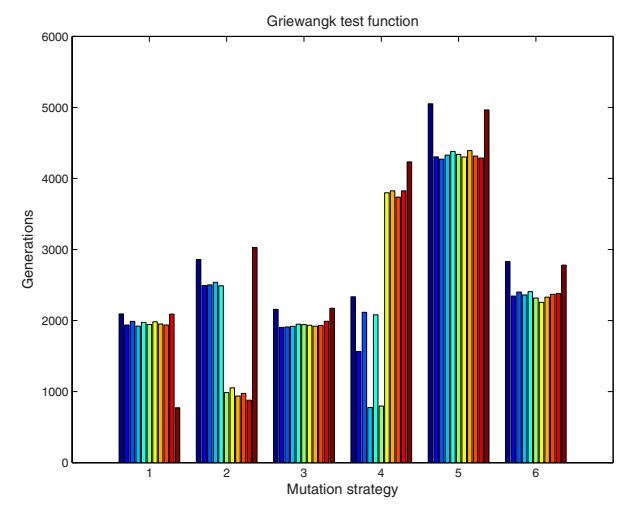

Fig. 5. Results for the Griewangk test function

A superior performance is typically obtained for intermediate values of $\varphi$. It has already been noted from the results of Table 2 and Figures 2 4 that the first mutation strategy, Equation (1), is the most efficient. From Figure 5 we can infer that this strategy also exhibits the most robust behavior with respect to the migration constant. The third mutation strategy, Equation (3), also exhibits a relatively robust behavior with respect to $\varphi$. It is worth noting, however, that the other considered mutation strategies can achieve a comparable, or even better, performance after fine-tuning the value of $\varphi$.

\subsection{Multiobjective Optimization}

Multiobjective Optimization (MO) problems consist of several competing and incommensurable objective functions. Such problems are frequently encountered in numerous scientific and engineering applications. The need for the concurrent minimization of more than one objective functions, renders the use of EAs particularly attractive. In contrast to traditional gradient-based techniques, EAs operate on a set of potential solutions of the problem. Thus, EAs are capable of detecting several solutions of an MO problem in a single run [11, 12, 42, 54, 59, 60]. These solutions are called Pareto optimal, and each corresponds to a different trade-off among the objective functions. Typically, a large number of Pareto optimal solutions exist.

Here we present a multi-population variant of DE, named Vector Evaluated Differential Evolution (VEDE), which is inspired by the Vector Evaluated Genetic Algorithm (VEGA) approach [42]. In VEDE, each population is evaluated using one of the objective functions of the problem under consideration. Information sharing among the populations takes place through the migration of the best individuals. The performance of a parallel version of VEDE, which incorporates a domination selection scheme, is investigated on widely used test problems and compared to the VEGA approach. The parallel computation of solutions of an MO problem is preferred, because besides the reduction in execution time, can also yield a better representation of the possible outcomes, thereby enhancing the performance of the algorithm [54]. 


\section{Background Material}

Let $\mathbb{S} \subset \mathbb{R}^{n}$ be an $n$-dimensional search space, and let $k$ objective functions:

$$
f_{i}(x): \mathbb{S} \rightarrow \mathbb{R}, \quad i=1,2, \ldots, k,
$$

be defined over $S$. Further assume, $g_{j}(x) \leqslant 0, \quad j=1, \ldots, m$, to be $m$ inequality constraints. Then the MO problem can be stated as finding a vector, $x^{*}=\left(x_{1}^{*}, x_{2}^{*}\right.$, $\left.\ldots, x_{n}^{*}\right)^{\top} \in S$, that satisfies the constraints and minimizes the function $\mathbf{f}(x)=$ $\left[f_{1}(x), f_{2}(x), \ldots, f_{k}(x)\right]: \mathbb{R}^{n} \rightarrow \mathbb{R}^{k}$. The goal of MO is to compute a set of Pareto optimal solutions to the aforementioned problem.

Let $u=\left(u_{1}, 2, \ldots, u_{k}\right)$, and $v=\left(v_{1}, v_{2}, \ldots, v_{k}\right)$, be two vectors. Then, $u$ dominates $v$ if and only if, $u_{i} \leqslant v_{i}, \quad i=1,2, \ldots, k$, and $u_{i}<v_{i}, \quad$ for at least one $i$. This property is known as Pareto dominance and it is used to define the Pareto optimal points. A solution, $x$, of the MO problem is said to be Pareto optimal if and only if, there does not exist another solution $y$, such that $\mathbf{f}(y)$ dominates $\mathbf{f}(x)$. The set of all Pareto optimal solutions of an MO problem is called Pareto optimal set and is denoted as $\mathcal{P}^{*}$. The set, $\mathcal{P} \mathcal{F}^{*}=\left\{\left(f_{1}(x), f_{2}(x), \ldots, f_{k}(x)\right)^{\top} \mid x \in \mathcal{P}^{*}\right\}$, is called Pareto front. A Pareto front $\mathcal{P F}^{*}$ is convex if and only if, there exists $w \in \mathcal{P} \mathcal{F}^{*}$, such that, $\lambda\|u\|+(1-\lambda)\|v\| \geqslant\|w\|, \quad \forall u, v \in \mathcal{P F}^{*}, \forall \lambda \in(0,1)$. Respectively, it is concave if and only if, there exists $w \in \mathcal{P F}^{*}$, such that, $\lambda\|u\|+(1-\lambda)\|v\| \leqslant\|w\|, \quad \forall u, v \in$ $\mathcal{P F}^{*}, \forall \lambda \in(0,1)$. A Pareto front can be convex, concave or partially convex and/or concave and/or discontinuous.

\section{The VEDE Algorithm}

For VEDE a number of $M$ subpopulations is considered in a prespecified ring topology. Each population is evaluated using as fitness function, one of the objective functions of the problem at hand. If $k$ is the number of the objective functions, and $k<M$, then the $i$-th population is evaluated according to the $j$-th objective function, where,

$$
j \equiv\left\{\begin{array}{ll}
i \bmod k, & \text { if } i \neq r k, \quad r=1,2, \ldots \\
k, & \text { otherwise }
\end{array} \text { and } i=1,2, \ldots, M .\right.
$$

In every generation, the best individual, $x_{g}^{\text {best }}$, of the $i$-th population, migrates to the $(i+1)$-th population of the ring. Then, the $(i+1)$-th population uses $x_{g}^{\text {best }}$ as the best individual to produce its mutant vectors at generation $(g+1)$. Obviously, only the DE operators that use the best individual in the mutations, i.e. the variants described in Equations (1), (3), and (4), can take full advantage of this information exchange procedure. Moreover, a domination selection procedure, similar to that of Abbass [2], is applied, i.e. instead of using the plain DE selection operator of Equation (7), we use the following one:

$$
x_{g+1}^{i}= \begin{cases}u_{g+1}^{i}, & \text { if } \mathbf{f}\left(u_{g+1}^{i}\right) \text { dominates } \mathbf{f}\left(x_{g}^{i}\right), \\ x_{g}^{i}, & \text { otherwise }\end{cases}
$$

where $\mathrm{f}$ is the vector function defined above. This selection scheme favors nondominated individuals in the population and it has proved to perform better in practice. VEDE can be easily parallelized. The populations can be distributed in several machines, with migrations taking place from node to node. 


\section{Experiments on Multi-Objective Optimization}

Four well-known MO benchmark problems were used in the investigation of VEDE's performance. Each test problem consists of two objective functions of the form

$$
\begin{aligned}
f_{1}\left(x_{1}\right) & =x_{1}, \\
f_{2}\left(x_{1}, x_{2}, \ldots, x_{n}\right) & =g\left(x_{2}, x_{3}, \ldots, x_{n}\right) \times h\left(f_{1}, g\right) .
\end{aligned}
$$

Specifically, we considered the following problems [60]:

Test Problem 1. This test problem is defined as:

$$
\begin{aligned}
f_{1}\left(x_{1}\right) & =x_{1}, \\
g\left(x_{2}, x_{3}, \ldots, x_{n}\right) & =1+\frac{9}{n-1} \sum_{i=2}^{n} x_{i}, \\
h\left(f_{1}, g\right) & =1-\sqrt{\frac{f_{1}}{g}}
\end{aligned}
$$

with $n=30$ and $x_{i} \in[0,1]$. The Pareto front for this problem is convex.

Test Problem 2. This test problem is the non-convex counterpart to Test Problem 1. It is defined as:

$$
\begin{aligned}
f_{1}\left(x_{1}\right) & =x_{1}, \\
g\left(x_{2}, x_{3}, \ldots, x_{n}\right) & =1+\frac{9}{n-1} \sum_{i=2}^{n} x_{i}, \\
h\left(f_{1}, g\right) & =1-\left(\frac{f_{1}}{g}\right)^{2},
\end{aligned}
$$

with $n=30$ and $x_{i} \in[0,1]$.

Test Problem 3. This test problem is defined as:

$$
\begin{aligned}
f_{1}\left(x_{1}\right) & =x_{1}, \\
g\left(x_{2}, x_{3}, \ldots, x_{n}\right) & =1+\frac{9}{n-1} \sum_{i=2}^{n} x_{i}, \\
h\left(f_{1}, g\right) & =1-\sqrt{\frac{f_{1}}{g}}-\frac{f_{1}}{g} \sin \left(10 \pi f_{1}\right),
\end{aligned}
$$

with $n=30$ and $x_{i} \in[0,1]$. The Pareto front consists of several convex parts.

Test Problem 4. This test problem is defined as:

$$
\begin{aligned}
f_{1}(x) & =x_{1}, \\
g\left(x_{2}, x_{3}, \ldots, x_{n}\right) & =1+10(n-1)+\sum_{i=2}^{n}\left(x_{i}^{2}-10 \cos \left(4 \pi x_{i}\right)\right), \\
h\left(f_{1}, g\right) & =1-\sqrt{\frac{f_{1}}{g}}
\end{aligned}
$$

and it has $21^{9}$ local Pareto fronts. 


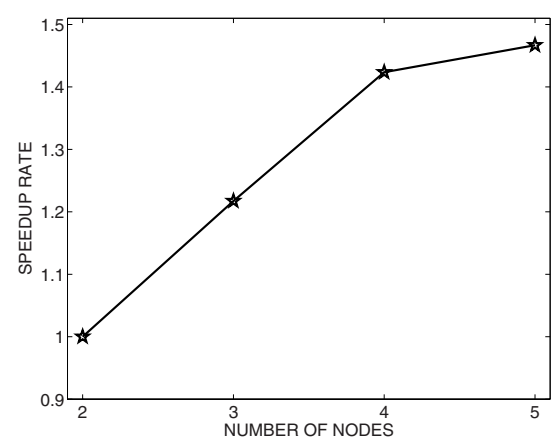

Fig. 6. Speedup gained using up to 5 nodes

All experiments were performed in parallel, using the PVM communication library. For the maintenance of the Pareto optimal set, the archiving technique described in [25], which uses an external archive, was employed. The obtained results were compared to that of VEGA algorithm. For this purpose, two established measures, namely the $\mathcal{C}$ measure [16, 60], and the $\mathcal{V}$ measure [16, 30] were employed. Metric $\mathcal{C}(A, B)$ measures the fraction of members of the Pareto front $B$ that are dominated by members of the Pareto front $A$, while $\mathcal{V}(A, B)$ is the fraction of the volume of the minimal hypercube containing both fronts, that is strictly dominated by members of $A$ but is not dominated by members of $B$ [16]. Following the analysis presented in [60], a total number of 100 individuals divided in several populations, as well as a maximum of 250 iterations per population per run, were used. We performed 30 experiments for each test problem, using the DE with the mutation operator of Equation (1), because it suits better the migration scheme described in the previous section. This variant is denoted as VEDE1. The results are reported in the boxplots of Figures. 7,10 .
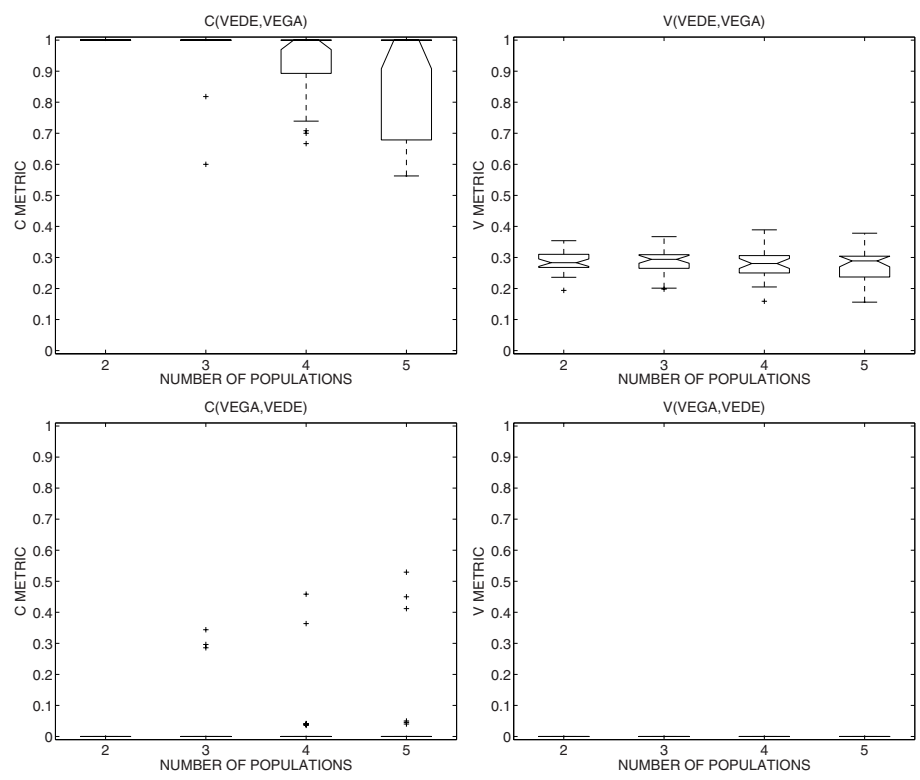

Fig. 7. Results of VEDE1 for the Test Problem 1 

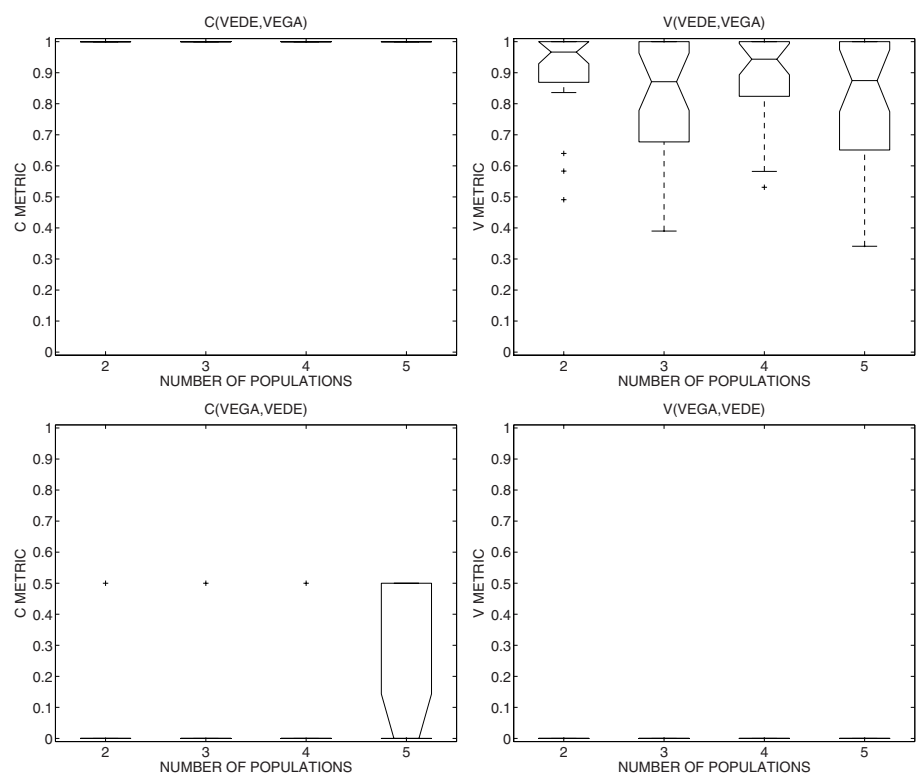

Fig. 8. Results of VEDE1 for the Test Problem 2
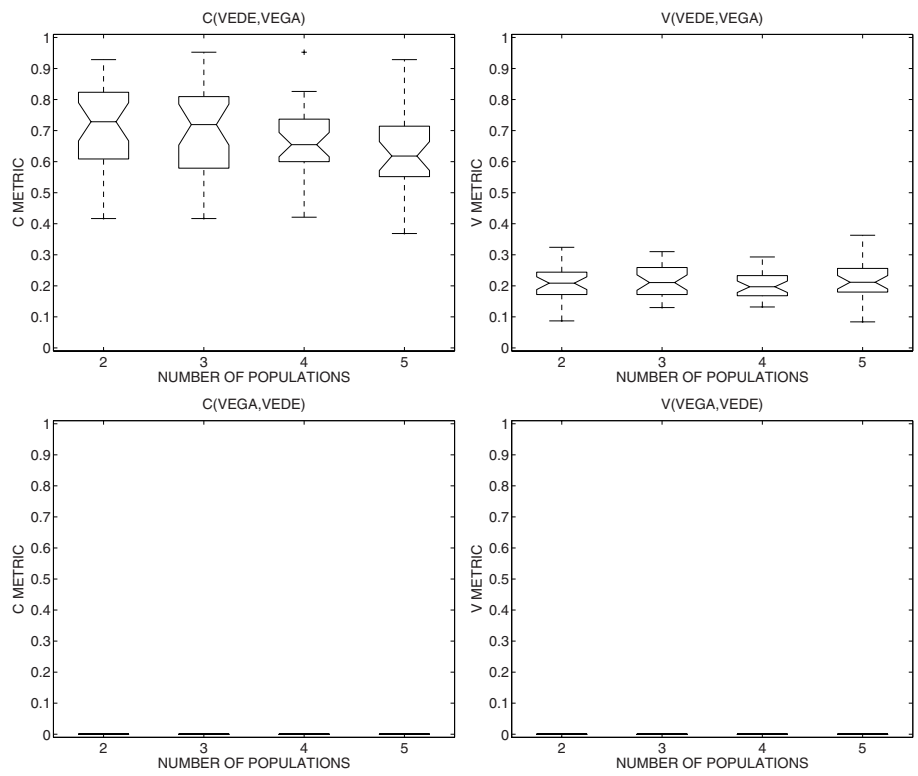

Fig. 9. Results of VEDE1 for the Test Problem 3 

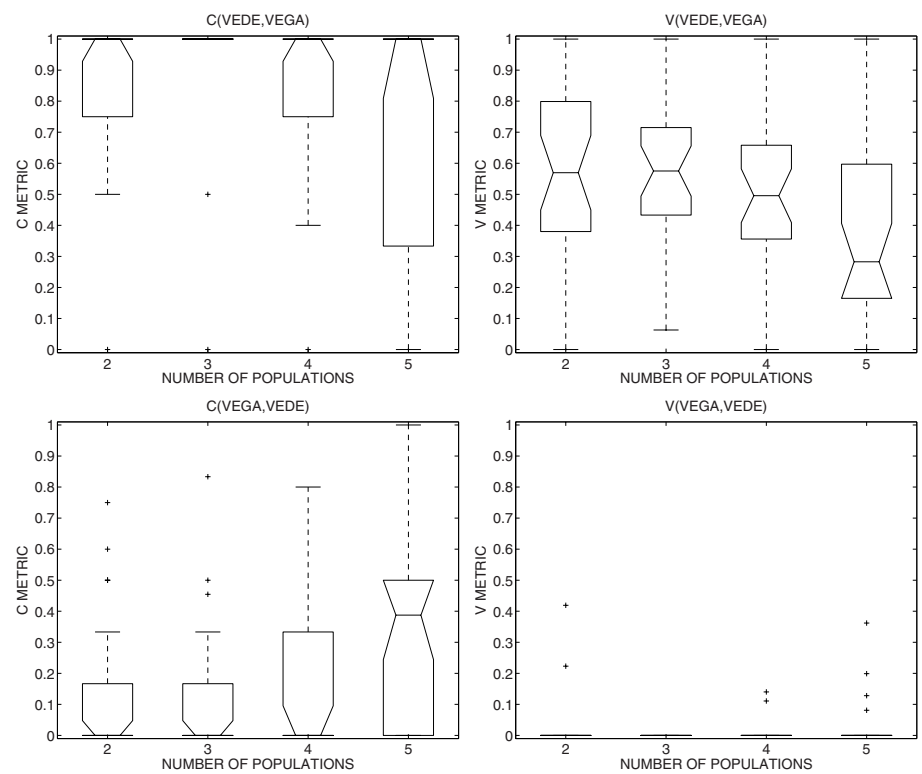

Fig. 10. Results of VEDE1 for the Test Problem 4

Each boxplot depicts the obtained values of the corresponding measure, in 30 experiments. The box has lines at the lower quartile, median, and upper quartile values. The lines extending from each end of the box (whiskers) show the extent of the rest of the data. The outliers, i.e. the values that lie beyond the ends of the whiskers, are denoted with crosses. DE is quite sensitive to population size, especially when the number of individuals becomes too small. This was verified in our preliminary experiments with VEDE. Dividing the 100 individuals into more than 5 populations (less than 20 individuals per population) resulted in substantial performance decline. Thus, our experiments were performed using 2 up to 5 populations. Standard values for the $\mu$ and $\rho$ parameters, equal to 0.7 and 0.9 , respectively, were used. The speedup gained from the parallel implementation using up to 5 nodes is depicted in Figure 6 As illustrated, there is an almost linearly increasing speedup rate using up to 4 nodes. Beyond 4 nodes, the speedup rate increases marginally. This effect can be attributed to the small number of individuals per population, which falls under 20. Additionally, we have tested the remaining DE mutation operators and in all cases, VEDE outperformed the VEGA with respect to the two metrics, $\mathcal{C}$ and $\mathcal{V}$. However, our results support the claim that VEDE, just like DE, is sensitive to population size.

\section{Computing Simultaneously Local and Global Minima}

In this Section the recently proposed clustering operator for Evolutionary Algorithms is described [48]. This operator utilizes already computed pieces of information regarding the search space in an attempt to discover regions containing groups of individuals 
located close to different minimizers. Consequently, the search is confined inside these regions and a large number of global and local minima of the objective function can be efficiently computed [48].

\subsection{Exploration vs. Exploitation}

The main problem when applying EAs is to find a set of control parameters which optimally balances the exploration and the exploitation capabilities of the algorithm. There is always a trade off between the efficient exploration of the search space and its effective exploitation. For example, if the recombination and mutation rates are too high, much of the space will be explored, but there is a high probability of losing good solutions. In extreme cases the algorithm has difficulty to converge to the global minimum due to insufficient exploitation of the search space. Fortunately, the convergence properties of the DE typically do not heavily depend on its control parameters. However, since not all search operators have the same impact on the exploration of the search space, the choice of the optimal mutation operator can be troublesome.

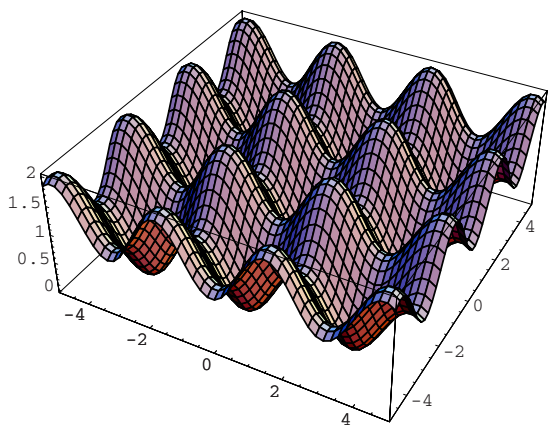

Fig. 11. Plot of $f(x)=\sin \left(x_{1}\right)^{2}+\sin \left(x_{2}\right)^{2}$

To illustrate this we utilize the simple multimodal 2-dimensional function: $f\left(x_{1}, x_{2}\right)=\sin \left(x_{1}\right)^{2}+\sin \left(x_{2}\right)^{2}$, where $\left(x_{1}, x_{2}\right) \in \mathbb{R}^{2}$. This function has an infinite number of global minimizers in $\mathbb{R}^{2}$, with function value equal to zero, at the points $(\kappa \pi, \lambda \pi)$, where $\kappa, \lambda \in \mathbb{Z}$. In the hypercube $[-5,5]^{2}$ the function $f$ has 9 global minimizers. In Figure 11 a surface plot of the function $f$ is exhibited. The six DE variants described above are applied to compute the global minimizers of the objective function $f$. Experimental results indicate that $\mathrm{DE}_{1}$ exhibits very fast convergence to one of the global minimizers of $f$. On the contrary, $\mathrm{DE}_{2}$ explores a large portion of the search space before converging to a solution. This behavior is illustrated in Figure 12, where (for visualization purposes) a population consisting of 1000 individuals is plotted after 1, 5, 10, 20 generations of $\mathrm{DE}_{2}$. A closer look at Equations (1) and (2) reveals that $\mathrm{DE}_{1}$ uses the best individual as a starting point for the computation of the mutant vector, thus constantly pushing the population closer to the location of the best computed point. On the other hand, since $\mathrm{DE}_{2}$ utilizes three randomly chosen individuals for the computation of the mutant one, its exploration capability is greatly enhanced. However, it exhibits lower convergence speed.

The performance of algorithms $\mathrm{DE}_{3}$ and $\mathrm{DE}_{4}$ resembles that of $\mathrm{DE}_{1}$, due to the use of the best individual. However, $\mathrm{DE}_{3}$ and $\mathrm{DE}_{4}$ exhibited better exploration than $\mathrm{DE}_{1}$, since they also incorporate randomly selected individuals. Algorithms $\mathrm{DE}_{5}$ and $\mathrm{DE}_{6}$ use only randomly selected individuals resulting in maximum exploration and the individuals of their populations are simultaneously attracted by more than one minimizers. 

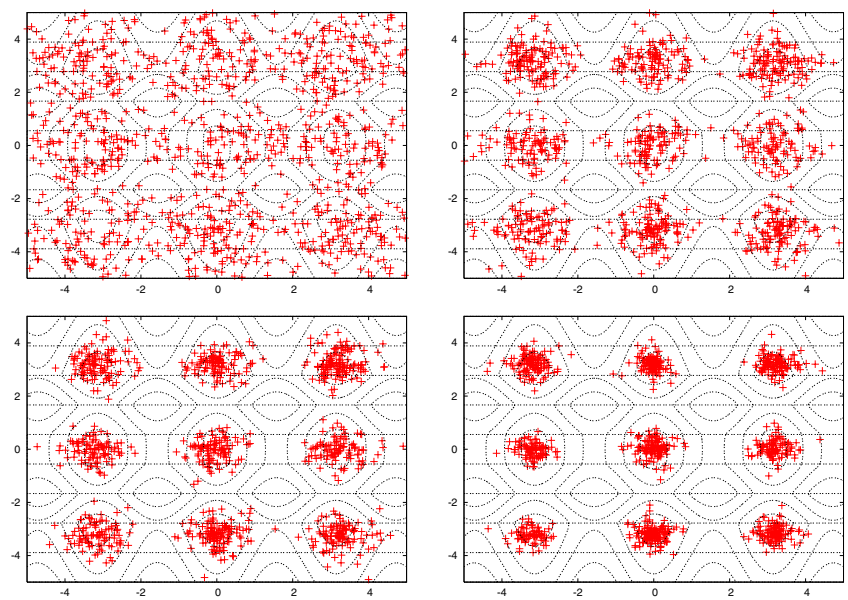

Fig. 12. $\mathrm{DE}_{2}$ population after $1,5,10$, and 20 generations

Experimental results show that some mutation operators have the tendency to concentrate subsets of the population in the region of attraction of different minimizers of the objective function. This observation motivated the incorporation of a clustering algorithm to identify such subsets. The $k$-windows clustering algorithm discovers boxes capturing subpopulations of individuals located in the region of a minimizer. Consequently, the subpopulations are confined to search within each box. Thus, the optimization of the objective function proceeds without affecting the dynamics of the DE algorithm. This process gives all the minimizers existing in regions that the DE algorithm explored before the use of the clustering operator. It is obvious that DE algorithm must adequately explore the search space prior to the call of the clustering operator. In the next section, for completeness purposes, we give a brief description of the $k$-windows clustering algorithm.

\subsection{The Unsupervised $k$-Windows Clustering Algorithm}

The recently proposed $k$-windows clustering algorithm [50] uses a windowing technique to discover the clusters present in an $n$-dimensional dataset. More specifically, assuming that the dataset lies in $n$ dimensions, the algorithm initializes a number of $n$-dimensional windows (boxes) over the dataset. Subsequently, it iteratively perturbs these windows using the movement and enlargement procedures, in order to capture within each window patterns that belong to a single cluster.

The movement and enlargement procedures are guided by the points that lie within each window. As soon as the movement and enlargement procedures do not significantly increase the number of points within each window they terminate. The final set of windows defines the clustering result of the algorithm.

A fundamental issue in cluster analysis, independent of the particular clustering technique applied, is the determination of the number of clusters present in a dataset. The 
unsupervised $k$-windows algorithm is capable to determine the number of clusters through a generalization of the original algorithm [50]. Finally, it must be noted that no objective function evaluations are necessary during the operation of the $k$-windows clustering algorithm [51].

\subsection{The Proposed Clustering Operator}

In this section, the clustering operator is described. This operator utilizes the unsupervised $k$-windows algorithm and is called only once, after a user-defined number of generations. In practice, a small number of generations is sufficient for the DE algorithm to explore the search space. Afterwards, the clusters of individuals are determined and subpopulations are confined within each region. Each subpopulation has $N P / \beta$ individuals, where $\beta$ is the number of clusters found. If a region contains more individuals, the clustering operator selects the best $N P / \beta$. On the other hand, if less individuals exist the clustering operator initializes new ones. The result of the algorithm is the location of many minimizers in a single run, including the global one.

To better utilize the described approach it is advisable to start the DE algorithm using a mutation operator that permits adequate exploration of the search space (for example $\mathrm{DE}_{2}$ or $\left.\mathrm{DE}_{6}\right)$. Once the clusters around the minima have been determined, one can switch to a mutation operator that has faster convergence speed (for example $\mathrm{DE}_{1}$ ).

For very hard optimization problems, when the objective function is defined in many dimensions and possesses multitudes of local and global minima, the clustering operator could be called more than once. The same might be true for real-life optimization tasks, where the function value of the global minimum is unknown.

Each consecutive call of the clustering

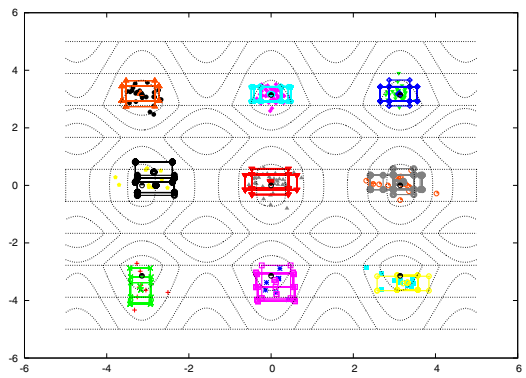

Fig. 13. Clusters and global minimizers operator will result in more promising subregions of the original search space and will save unneeded objective function evaluations, since the subpopulations will stay focused on regions containing desirable minimizers. For all the experiments reported here, one call of the clustering operator was sufficient for the algorithm to locate the global, as well as, many local minimizers. To determine the applicability and the efficiency of the clustering operator we incorporated it to the DE algorithm and applied the new method to the multimodal test function $f$, which posses 9 global minimizers in the hypercube $[-5,5]^{2}$. The result of the application of the clustering operator on the function $f$ is illustrated in Figure 13

It must be noted that a number of independent experiments of the original DE algorithm gives no guarantee that all global minimizers will be detected, since the algorithm has no memory; no information concerning previously detected minimizers is kept. We performed 100 independent simulations, using each one of the six different mutation operators described above and Table 3 exhibits the average number of restarts needed 
Table 3. Restarts needed to locate all the minimizers of $f$

\begin{tabular}{cccccccc}
\hline & \multicolumn{3}{c}{ Original DE Algorithm } & & \multicolumn{3}{c}{ DE with $k$-win } \\
\cline { 2 - 4 } \cline { 7 - 8 } & Min & Mean & Max & & Min & Mean & Max \\
\hline $\mathrm{DE}_{1}$ & 54 & 110.2 & 203 & & 1 & 17.5 & 66 \\
$\mathrm{DE}_{2}$ & 57 & 119.1 & 294 & & 1 & 1.0 & 1 \\
$\mathrm{DE}_{3}$ & 60 & 133.0 & 239 & & 1 & 1.1 & 7 \\
$\mathrm{DE}_{4}$ & 52 & 114.9 & 212 & & 1 & 1.0 & 1 \\
$\mathrm{DE}_{5}$ & 49 & 106.7 & 245 & & 1 & 1.0 & 1 \\
$\mathrm{DE}_{6}$ & 62 & 111.4 & 221 & & 1 & 1.0 & 1 \\
\hline
\end{tabular}

for the DE algorithms to locate all the global minimizers of $f$. The modified algorithm that uses the clustering operator in most cases managed to find all the minimizers of $f$ in a single execution.

\subsection{Experimental Results on Multi-minima Discovery}

We implemented and tested the clustering operator on a number of hard optimization tasks and it exhibited stable and robust performance. We report results from the Levy No. 5 test function. For each mutation operator we performed 100 independent experiments. A population consisting of 200 individuals was used and the mutation and recombination constants had values $\mu=0.6$ and $\rho=0.8$, respectively. The algorithm was terminated when the global minimum was located. The clustering operator was called only once for the optimization of each of the four test function considered below, after 20, 20, 10, and 200 generations, respectively. Table 4 summarizes the average results for the 100 runs. The first column of the table indicates the name of the algorithm and the second column the average number of generations needed for the algorithm to locate the global minimum without the use of the clustering operator. The third and fourth columns give the average number of minimizers discovered (including the global one) and the corresponding average generations needed for the DE algorithm to locate the global minimum using the clustering operator.

The Levy No. 5 test function is given by the following equation:

$$
f_{1}(\mathbf{x})=\sigma_{1} \sigma_{2}+\left(x_{1}+1.42513\right)^{2}+\left(x_{2}+0.80032\right)^{2},
$$

where $x_{i} \in[-10,10], i=1,2$, and $\sigma_{1}$ and $\sigma_{2}$ are given by:

$$
\sigma_{1}=\sum_{i=1}^{5} i \cos \left[(i+1) x_{1}+i\right], \text { and } \sigma_{2}=\sum_{j=1}^{5} j \cos \left[(j+1) x_{2}+j\right] .
$$

There exist about 760 local minima and one global minimum with function value $f_{1}^{*}=$ -176.1375 located at $\mathrm{x}^{*}=(-1.3068,-1.4248)$. The large number of local optimizers makes extremely difficult for any method to locate the global minimizer.

The experimental results exhibited in Table 4 indicate that generally the use of the clustering operator enhances the performance of the DE algorithms. In detail, there is an average acceleration of the algorithm's convergence speed ranging from $30 \%$ to $80 \%$. 
Table 4. Average results for the Levy function

\begin{tabular}{ccccc}
\hline & w/o $k$-win operator & & \multicolumn{2}{c}{ with $k$-win operator } \\
\cline { 2 - 2 } & Generations & & Minima located & Generations \\
\hline $\mathrm{DE}_{1}$ & 33.21 & & 5.97 & 34.36 \\
$\mathrm{DE}_{2}$ & 70.66 & & 20.52 & 54.26 \\
$\mathrm{DE}_{3}$ & 64.09 & & 11.96 & 39.77 \\
$\mathrm{DE}_{4}$ & 65.11 & & 20.22 & 50.25 \\
$\mathrm{DE}_{5}$ & 133.01 & & 22.70 & 70.85 \\
$\mathrm{DE}_{6}$ & 64.89 & & 19.20 & 50.24 \\
\hline
\end{tabular}

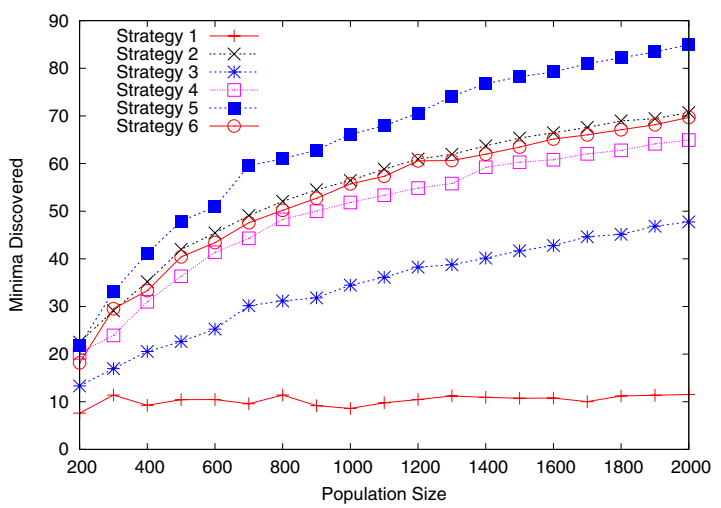

Fig. 14. The effect of the population size on the number of located minimizers

Additionally, as many as 20 minimizers (including the global one) were simultaneously computed. The only exception is $\mathrm{DE}_{1}$ where a slight increase in the generations is observed ( $3 \%$ ), but the modified algorithm locates the global as well as 5 local minimizers.

To better demonstrate the ability of this approach to locate many minima at once, independent runs were conducted with the number of individuals in the population gradually increasing from 200 to 2000. In general, a larger population explores better the search space and more regions containing minimizers are located by the $k$-windows operator. In Figure 14 we exhibit the detailed results. The algorithm $\mathrm{DE}_{1}$ locates on average 10 minimizers regardless of the size of its populations. On the contrary, the rest of the algorithms locate more minimizers as their population is increased. $\mathrm{DE}_{5}$ exhibited the best performance finding simultaneously up to 85 minimizers.

The above experiments show that this approach greatly accelerates the convergence speed of the DE algorithms and that in addition to the global minimum is capable to locate simultaneously many local minima without extra function evaluations. To this end, the use of the clustering operator is always suggested. In brief, the clustering operator has the following advantages:

1. locates global minimizers and local minimizers with relatively low function value,

2. in general, fewer generations are required for the DE algorithm to converge,

3. there is no need for additional function evaluations, 
4. utilizes the range search algorithm for fast and reliable execution,

5. its parallel implementation is straightforward,

6. is better suited to difficult high-dimensional multimodal objective functions.

\section{Neural Network Training Using DE}

Artificial Feedforward Neural Networks (FNNs) have been widely used in many application areas in recent years and have shown their strength in solving hard problems in Artificial Intelligence. Although many different models of neural networks have been proposed, multilayered FNNs are the most common. FNNs consist of many interconnected identical simple processing units, called neurons. Each neuron calculates the dot product of the incoming signals with its weights, adds the bias to the resultant, and passes the calculated sum through its activation function. In a multilayer feedforward network the neurons are organized into layers with no feedback connections [23].

The incremental adaptation of the connection weights that propagate information between the neurons, is called training. The majority of the training algorithms use the negative of the gradient of the error function, $-\nabla E(w)$, as their descent direction. The gradient $\nabla E(w)$ can be computed by the BackPropagation of the error through the layers of the network. Here, a new class of DE-based training algorithms that do not need the gradient of $E$ and train integer weight neural networks with threshold units is discussed. Formally, a typical FNN consists of $L$ layers, where the first layer denotes the input, the last one, $L$, is the output, and the intermediate layers are the hidden layers. It is assumed that the $(l-1)$ layer has $N_{l-1}$ neurons. The neurons operate according to the following equations

$$
n e t_{j}^{l}=\sum_{i=1}^{N_{l-1}} w_{i j}^{l-1, l} y_{i}^{l-1}+\theta_{j}^{l}, \quad y_{j}^{l}=f^{l}\left(\text { net }_{j}^{l}\right),
$$

where $w_{i j}^{l-1, l}$ is the integer connection weight from the $i$-th neuron at the $(l-1)$ layer to the $j$-th neuron at the $l$-th layer, $y_{i}^{l}$ is the output of the $i$-th neuron belonging to the $l$-th layer, $\theta_{j}^{l}$ denotes the integer bias of the $j$-th neuron at the $l$-th layer, and $f$ is the activation function. The weights in the FNN can be expressed in vector notation. Let the weight vector have the form: $w=\left(w_{1}, w_{2}, \ldots, w_{n}\right)$. The weight vector, in general, defines a point in the $n$-dimensional real Euclidean space $\mathbb{R}^{n}$, where $n$ denotes the total number of weights and biases in the network. From the optimization point of view, supervised training of an FNN is equivalent to minimizing the corresponding error function, which is a multivariate function that depends on the weights in the network. The square error over the set of input-desired output patterns with respect to every weight, is usually taken as the function to be minimized. Specifically, the error function for an input pattern $t$ is defined as, $e_{j}(t)=y_{j}^{L}(t)-d_{j}(t), j=1,2, \ldots, N_{L}$, where $d_{j}(t)$ is the desired response of an output neuron at the input pattern $t$. For a fixed, finite set of input-desired output patterns, the square error over the training set which contains $T$ representative pairs is:

$$
E(w)=\sum_{t=1}^{T} E_{t}(w)=\sum_{t=1}^{T} \sum_{j=1}^{N_{L}} e_{j}^{2}(t),
$$


where $E_{t}(w)$ is the sum of the squares of errors associated with the pattern $t$. Minimization of $E$ is attempted by using a training algorithm to update the weights.

\subsection{Training Integer Weight Neural Networks with Threshold Activations}

FNNs can be simulated in software, but to be utilized in real life applications, where high speed of execution is required, hardware implementation is needed. The natural implementation of an FNN - because of its modularity - is a parallel one. Hardwarefriendly algorithms are essential to ensure the functionality and cost effectiveness of the hardware implementation. Moreover, the need for hardware-friendly algorithms, which have the ability to cope with time-varying problems and real-time timing constraints, has been recently increased [35].

FNNs having integer weights and biases are easier and less expensive to implement in electronics as well as in optics and the storage of the integer weights is much easier to be achieved. Additionally, the use of threshold activation functions for all the hidden and output neurons, greatly reduces the complexity of the hardware implementation, because there is no need to design and implement complicated non-linear activation functions. Another advantage of the FNNs with integer weights and threshold activation functions is that the trained neural network is to some extend immune to noise in the training data. Such networks only capture the main feature of the training data. Low amplitude noise that possibly contaminates the training set cannot perturb the discrete weights, because those networks require relatively large variations to "jump" from one integer weight value to another.

Mathematical operations that are easy to implement in software might often be very burdensome in the hardware and therefore more costly. This property reduces the amount of memory required for weight storage in digital electronic implementations. Additionally, it simplifies the digital multiplication operation. Finally, if inputs are restricted to the set $\{-1,1\}$ (bipolar inputs), the neurons in the first hidden layer require only sign changes during multiplication operations, and only integer additions.

To apply DE to neural network training with integer weights, we initialize the individuals with $N$-dimensional integer weight vectors, following a uniform probability distribution, and evolve them over time. The only problem is that the mutation operator results in real weight vectors. As our aim is to maintain an integer weight population at each generation, each component of the mutant weight vector is rounded to the nearest integer.

It must be noted that the evolutionary class of algorithms does not need the activation function to be differentiable and is suitable for training with threshold units [34]. In the first phase of our approach, the DE algorithms are used to train a neural network "offline", using sigmoid activation functions, such as:

$$
f_{1}(x)=\tanh (\lambda x) \equiv \frac{2}{1+e^{-\lambda x}}-1, \quad f_{2}(x)=\frac{1}{1+e^{-\lambda x}},
$$

where $\lambda$ is the gain parameter. This seems to be a good practice since the network is trained much faster with sigmoid functions. In the second phase we alter the gain of the sigmoid function in such a way that allows a mapping to a threshold unit network. 
Specifically, when the inputs are correctly classified and the network error is relatively small, the value of $\lambda$ is increased in the sequence $(1,10,20,30,40,50, \infty)$. Additional training might be necessary after each increase of $\lambda$. That justifies the additional iterations needed to train an FNN, using only threshold activation functions. This procedure is analogous to taking the limit of the sigmoid function as the gain parameter $\lambda$ goes to infinity. Finally, the trained network uses only threshold activation functions and thus the complexity of the hardware implementation is greatly reduced. If new input data are introduced, training can be continued sequentially or in parallel "on-chip".

\subsection{Experiments on Neural Network Training}

Next, we exhibit results of the DE-based algorithms on the Encoder/Decoder FNN training problem. For all the simulations bipolar input and output vectors have been used. Table 5 summarizes the performance of the DE algorithms using different mutation rules when sigmoid activation functions are used. Hyperbolic tangent activation functions in both the hidden and output layer neurons have been used. In Table 6 we exhibit the performance of the DE algorithms, when the training has been performed as described above in order to lead to a network that uses only threshold activation functions.

We must note here that a key feature of the DE algorithms is that only error function values are needed. No gradient information is required, so there is no need of backward passes. For the test problems considered, we made no effort to tune the mutation, recombination and migration constants, $\mu, \rho$ and $\varphi$ respectively, to obtain optimal or at least nearly optimal convergence speed. Default fixed values $(\mu=0.5, \rho=0.7$ and $\varphi=0.3$ ) have been used instead. Smaller values of $\varphi$ can further reduce the messages between the processors, but may result in rare and inefficient migrations. It is obvious that one can try to fine-tune the $\mu, \rho, \varphi$ and $N P$ parameters to achieve better results, i.e. less error function evaluations and/or exhibit higher success rates. The weight subpopulations have been initialized with random integers from the interval $[-3,3]$ and the total population size $3 N P$ has been divided equally to 3 subpopulations, each having $N P$ individuals. Regarding the total population size, experimental results have shown that a good choice is $2 n \leqslant 3 N P \leqslant 4 n$, where $n$ denotes the dimensionality of the error function, i.e. the total number of weights and biases. It is obvious that the exploitation of the weight space is more effective for large values of $N P$, but sometimes more error function evaluations are required. On the other hand, small values of $N P$ render the algorithm inefficient and more generations are required to converge.

\section{4-2-4 Encoder/Decoder}

Here, we consider the 4-2-4 encoder/decoder (sixteen weights and six biases, dimension of the problem $n=22$ ). The network is presented with 4 distinct input patterns, each having only one bit turned on. The task is to duplicate the input pattern in the output units. Since all information must flow through the hidden units, the network must develop a unique encoding for each of the 4 patterns in the 2 hidden units and a set of connection weights performing the encoding and decoding operations. This particular encoding is considered to be "tight", since the number of the hidden nodes equals the 
Table 5. Results for the encoder/decoder problem using sigmoid activation functions

\begin{tabular}{crrrrc}
\hline Algorithm & \multicolumn{1}{c}{ min } & \multicolumn{1}{c}{ mean } & \multicolumn{1}{c}{$\max$} & \multicolumn{1}{c}{ s.d. } & Success \\
\hline $\mathrm{DE}_{1}$ & 330 & 1614.8 & 4686 & 868.5 & $100 \%$ \\
$\mathrm{DE}_{2}$ & 3960 & 8160.6 & 13376 & 2160.5 & $100 \%$ \\
$\mathrm{DE}_{3}$ & 308 & 1428.2 & 4004 & 660.9 & $100 \%$ \\
$\mathrm{DE}_{4}$ & 660 & 4540.5 & 8514 & 1505.4 & $100 \%$ \\
$\mathrm{DE}_{5}$ & 7260 & 13110.9 & 20636 & 3092.4 & $100 \%$ \\
\hline
\end{tabular}

Table 6. Results for the encoder/decoder problem using threshold activation functions

\begin{tabular}{ccrrrc}
\hline Algorithm & min & \multicolumn{1}{c}{ mean } & \multicolumn{1}{c}{$\max$} & \multicolumn{1}{c}{ s.d. } & Success \\
\hline DE $_{1}$ & 990 & 2520.8 & 23260 & 2326.4 & $100 \%$ \\
$\mathrm{DE}_{2}$ & 4796 & 8724.9 & 16588 & 2264.6 & $100 \%$ \\
$\mathrm{DE}_{3}$ & 1034 & 2104.5 & 4664 & 680.0 & $100 \%$ \\
$\mathrm{DE}_{4}$ & 1870 & 4778.1 & 9724 & 1278.0 & $100 \%$ \\
$\mathrm{DE}_{5}$ & 6072 & 14070.3 & 20746 & 2795.4 & $100 \%$ \\
\hline
\end{tabular}

base 2 logarithm of the input nodes $\left(\log _{2} 4=2\right)$. This problem has been selected because it is quite close to real world pattern classification tasks, where small changes in the input pattern cause small changes in the output pattern. The size of each subpopulation was $N P=20$. The low and high bound of the age of each individual, were $\alpha=50$ and $\beta=200$ respectively. A typical 3-bit weight vector is $w=(0,2,-2,3,-3,-3$, $2,3,-3,-3,2,-3,-2,2,3,2,1,0,-3,-3,-2,-2)$ and the corresponding value of the error function is $E=0.0459$. Simulation results are exhibited in Tables 5 and 6

\section{Data Mining Using DE}

Although originally designed for global optimization the DE algorithm is versatile enough to be applied in a variety of scientific tasks. For example here we present an application of DE for data clustering. Evolutionary clustering is a recent trend in cluster analysis, that has the potential to yield high partitioning accuracy results. Traditional evolutionary techniques applied in clustering are typically hindered by the high cost involved in the computation of the objective function. In this section we demonstrate how DE can be employed to evolve cluster solutions. Furthermore we present how recent advances in clustering can be employed to estimate the number of clusters from this evolutionary technique. Finally, by employing real world datasets, we exhibit the high quality clustering results that this scheme can provide.

\subsection{Data Clustering}

Clustering is a fundamental step in the process of transforming data to knowledge. It aims at discovering groups (clusters) in a set of objects such that similarity among the objects in the same group is higher than that of objects belonging to different clusters. 
The application domain of clustering techniques is very wide including data mining, text mining, statistical data analysis, compression and vector quantization, global optimization and web personalization [7, 15, 38, 52].

Clustering algorithms are traditionally categorized into three main categories, Hierarchical, Partitioning [44] and Distance-based. Hierarchical clustering algorithms construct hierarchies of clusters in a top-down (agglomerative) or bottom-up (divisive) fashion. Hierarchical clustering algorithms have proved to yield high quality results especially for applications involving clustering text collections. Nonetheless, their high computational requirements, usually limits their applicability in real life applications, where the number of samples and their dimensionality is typically high (the cost is quadratic to the number of samples).

Partitioning clustering algorithms, start from an initial clustering (that can be randomly formed) and create partitionings by iteratively adjusting the clusters based on the distance of the data points from a representative member of the cluster. The most commonly used partitioning clustering algorithm is $k$-means. This algorithm initializes $k$ centers and iteratively assigns each data point to the cluster whose centroid minimizes the Euclidean distance from the data point. Algorithms of this type can give good clustering results at low cost, since their running time is proportional to $k N$, where $N$ is the number of patterns present in the dataset. However, their results rely heavily on their initialization and they can converge to arbitrary local optima.

Distance based clustering algorithms create a partitioning by considering neighbors of data points. DBSCAN [41] is a distance-based clustering algorithm that has proved quite effective for spatial databases. Clusters are considered as high density neighborhoods of data points. Although the density parameter is critical for the successful application of DBSCAN, recently proposed heuristics appear to yield high quality results. The computational complexity of DBSCAN comes up to $O(N \log (N))$ under the assumption that the data are organized in a spatial index ( $R^{*}$-tree).

In evolutionary clustering, a solution to the clustering problem is typically encoded as a chromosome. By employing evolutionary operators and a population of solutions the algorithm probes the search space to find a globally optimum partition of the data. In early approaches [9, 26], chromosomes encoded the partition of $n$ objects into $K$ clusters and Genetic Algorithms were employed to identify the best partition. However, the sensitivity of GAs to the selection of the various parameters like population size, and crossover and mutation probabilities, as well as, the difficulties associated with the representation scheme, presented a major problem. Better results were obtained through hybrid approaches [5].

However, it is possible to represent the clustering procedure as an optimization problem of locating the optimal centroids of clusters. Thus, all evolutionary techniques can be employed since a clustering solution can be represented as a real-valued vector of the centroids. Previous approaches employed Evolutionary Strategies [6], Evolutionary Programming [18], and recently Particle Swarm Optimization [53]. All these approaches demonstrated that it is possible to obtain high quality partitions, but at a high computational cost. Here we attempt to tackle the high computational cost of traditional evolutionary techniques by introducing a new fitness criterion. This criterion is based on a windowing technique already employed in other clustering algorithms [51, 55]. 
A critical and open issue in cluster analysis, is the determination of the number of clusters present in a dataset. The evolutionary clustering techniques proposed so far, with the exception of [22], require from the user to specify the number of clusters present in the data prior to the execution of the algorithm. The described approach can provide an approximation to the number of clusters present in a dataset.

\subsection{Designing an Efficient Clustering Fitness Criterion}

Let the data set comprise a set $X=\left\{x_{1}, x_{2}, \ldots, x_{n}\right\}$, where $x_{j}$ is a data vector in the $d$ dimensional Euclidean space $\mathbb{R}^{d}$. A $k$ clustering of $X$ is a partition $C$ of $X$ into $k$ disjoint groups $C_{i}$, for $i=1,2, \ldots, k$. The clustering problem amounts to the determination of a partition of $X$ which is optimal with respect to a function $f$ that quantifies the goodness of the partition.

Different statistical functions have been proposed for $f$ [31, 58]. But in most approaches at least a full scan over the dataset is necessary to compute the function value for a specific instance. Evolving a population using such a fitness criterion can be expensive in terms of computational cost, compared to $k$-means like approaches that typically do not require more than 10 to 20 scans of the dataset. However an efficient clustering fitness criterion can be constructed by utilizing computational geometry techniques. In detail, let us define structure in the form of axis parallel hyper-rectangles ( $d$-ranges).

Definition 1: Let a $d$-range of size $a \in \mathbb{R}$ and center $z \in \mathbb{R}^{d}$ be the orthogonal range $\left[z_{1}-a, z_{1}+a\right] \times \cdots \times\left[z_{d}-a, z_{d}+a\right]$. Assume further, that the set $S_{a, z}$, with respect to the set $X$, is defined as $S_{a, z}=\left\{y \in X: z_{i}-a \leqslant y_{i} \leqslant z_{i}+a, \quad \forall i=1,2, \ldots, d\right\}$. Then the Window Density Function WDF for the set $X$, with respect to a given size $a \in \mathbb{R}$, is defined as: $\mathrm{WDF}_{a}(z)=\left|S_{a, z}\right|$, where $|\cdot|$ denotes the cardinality of the set, i.e. a measure of the number of elements of the set.

In other words, WDF represents the number of points from the dataset $X$, that reside in a window of size $a$ centered at $z$. WDF is a meaningful clustering objective function, since as the center of a $d$-range, $z$, moves to the center of the cluster the number of points around it should increase. As it is obvious the size $a$, is critical to the procedure as it

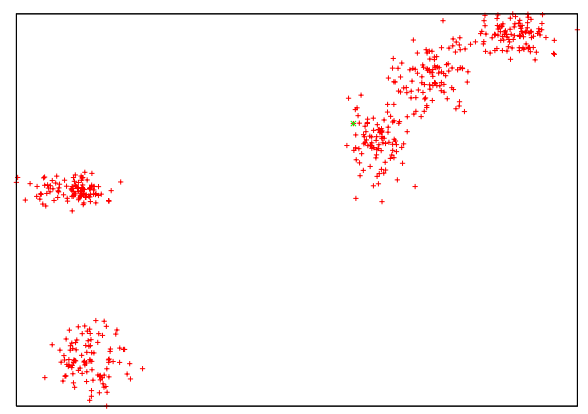

Fig. 15. Dataset $D S e t_{1}$ 

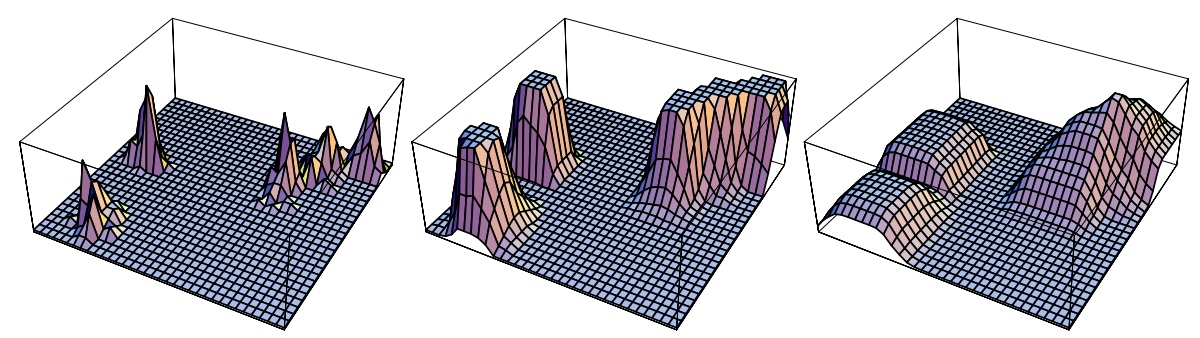

Fig. 16. 3D-Plot of WDF for Dset $_{1}$ and $a=1,5,10$ (left to right)

determines the location of the minimizers of the objective function. To illustrate this we employ the dataset Dset $_{1}$, exhibited in Figure 15 This dataset contains 500 points organized in 5 clusters with 100 points each. Each cluster is constructed by sampling 100 points from a two dimensional Gaussian distribution. The mean of each distribution was randomly scattered in the range $[0,200]^{2}$, and the covariance matrices were randomly generated by obtaining for each element of the matrix a random number between 1 and 2 .

In Figure 16, the 3D plots of WDF are provided to visualize the impact of the parameter $a$. As the value of $a$ increases, the extreme points of WDF tend to merge. When $a=1$ there are five maxima, equal to the number of clusters. On the other hand, when $a=10$, the three maxima corresponding to the three closest clusters previously identified merge to a single one.

The most important feature of the density function is that it is not necessary to scan the entire dataset to obtain a fitness value for a specific object. In particular, the computation of WDF is the well studied Computational Geometry Orthogonal Range Search Problem. Numerous Computational Geometry techniques have been proposed to address this problem. All these techniques employ a preprocessing stage at which they construct a data structure storing the patterns. This data structure allows them to answer range queries fast. For applications of very high dimensionality, data structures like the Multidimensional Binary Tree [37], and Bentley and Maurer [8] seem more suitable. On the other hand, for low dimensional data with a large number of points the approach of Alevizos [3] appears more attractive.

\subsection{Evolutionary Clustering under the WDF Objective Function}

In the DE settings, the population of potential solutions should be properly defined to represent nominal clustering solutions. As such, each individual, in the clustering context, is expressed using a predetermined number of $d$-dimensional vectors that represent the centers of the $d$-ranges, which in turn constitute the clustering result. The fitness of each individual is measured by the sum of the WDF function over all the $d$-ranges, under a fixed value of the parameter $a$. The remaining procedure of the DE algorithm remains unchanged.

As it is obvious an evolutionary optimization procedure using the above described characteristics aims at discovering the set of $d$-ranges that include as many points from 
(a)

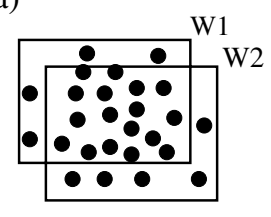

(b)

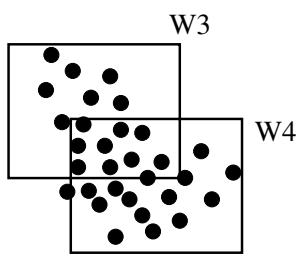

(c)

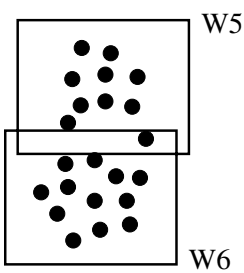

Fig. 17. (a) $W 1$ and $W 2$ satisfy the similarity condition and $W 1$ is deleted. (b) $W 3$ and $W 4$ satisfy the merge operation and are considered to belong to the same cluster. (c) $W 5$ and $W 6$ capture two different clusters.

the dataset as possible. Thus a single execution is able to determine a clustering result. Note that in the final clustering solution empty $d$-ranges may appear, or even $d$ ranges that overlap may exist. By employing the merge operation of the unsupervised $k$-windows clustering algorithm [51], the number of clusters can be approximated. During this step, for each pair of overlapping windows, the number of patterns that lie in their intersection is computed. With respect to the proportion of this number to the total number of points contained in each window, the algorithm can decide whether to either:

(a) Ignore one window if the proportion is very high.

(b) Consider the windows to contain parts of the same cluster if the proportion is relatively high.

(c) Consider the windows to capture different clusters, if the proportion is low.

An example of this operation is exhibited in Figure 17 and a high level description of the described algorithmic scheme follows:

\section{DEEC algorithm}

Step 1. Construct a data structure for the storing of the data.

Step 2. Set the parameter $a$ of WDF function.

\section{Step 3. Repeat}

Step 4. Execute the DE algorithm.

Step 5. Until a sufficient part of the dataset is covered or a maximum number or iterations is performed.

Step 6. Merge the resulting $d$-ranges

Step 7. Report the final clusters.

\subsection{Evolutionary Clustering Results}

To demonstrate the applicability of this approach we firstly employ Dset $_{1}$, exhibited in Figure 15, which is two dimensional and allows the visual inspection of the results. Note that in all the experiments reported in this section the population size was set to 20 individuals, and a maximum of 200 epochs was allowed. The DE parameters $\mu$ and $p$ were set to 0.6 , and 0.8 , respectively, in all experiments. Moreover, if the $d$-ranges of the best individual discovered contain more than $90 \%$ of the total points the execution 

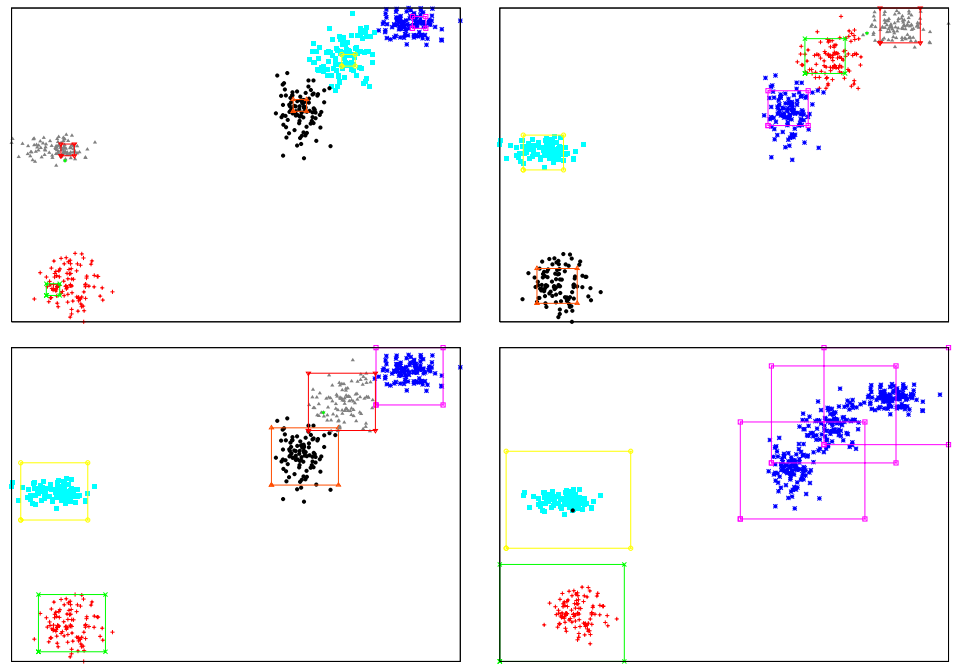

Fig. 18. The clustering result of DEUC for $a=1,3,5,10$

of DE terminates. The application of the DEUC algorithm over the Dset $_{1}$ dataset with the parameter $a$ obtaining values 1, 2, 5, 10 is exhibited in Figure 18 These results were obtained, by stopping the iterative executions of DE when more than $90 \%$ of the dataset was covered. Each individual encoded the center of five $d$-ranges. Comparing the clustering result, with the 3D plots of the WDF function in Figure 16, it is obvious that DEUC is able to detect the extrema of WDF and form a clustering result that is in accordance with the form of WDF. The colors in the plots correspond to the different cluster labels of the points that were assigned to the closest $d$-range under the Euclidean metric. It is obvious that DEUC is able to provide visually optimal clustering results when $a$ ranges between 1 and 5 . On the other hand, when $a$ is too large the adjacent clusters are merged to a single cluster by the merging procedure.

Comparing the results of DEUC involves the usage of a clustering algorithm that can approximate the number of clusters. To compare the results of DEUC with other approaches we employ the DBSCAN clustering algorithm [41]. This choice is motivated by the fact that DBSCAN computes the number of points (MinPts) that reside in a hypersphere of size Eps. Thus, the Eps parameter of DBSCAN is strongly related to the $a$ parameter of the WDF function. The execution of DBSCAN on Dset $_{1}$, setting MinPts $=5$, (anything with less than 5 points in an Eps neighborhood around it is considered noise), and for Eps obtaining the values Eps $=1,3,5,10$ is exhibited in Figure 19. Similarly in this case the colors designate different cluster labels, and the red crosses represent points recognized as noise. From the plots we can see that DBSCAN is more sensitive to the value of Eps than DEUC is on the value of $a$. Moreover, for DBSCAN to be able to recognize the three different adjacent clusters a very careful selection of Eps and MinPts is needed. 

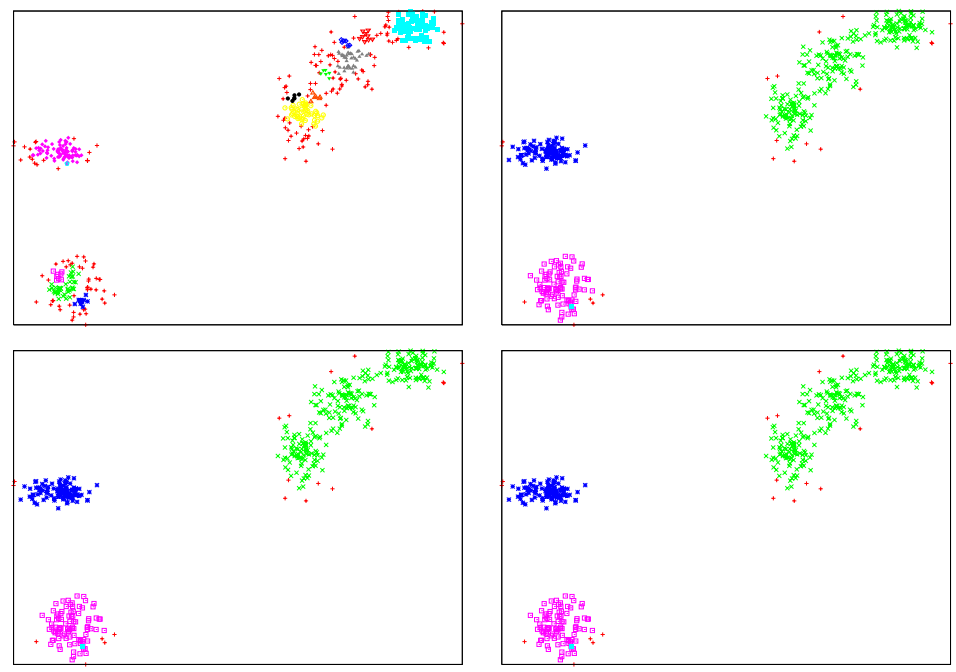

Fig. 19. The clustering result of DBSCAN for Eps $=1,3,5,10$
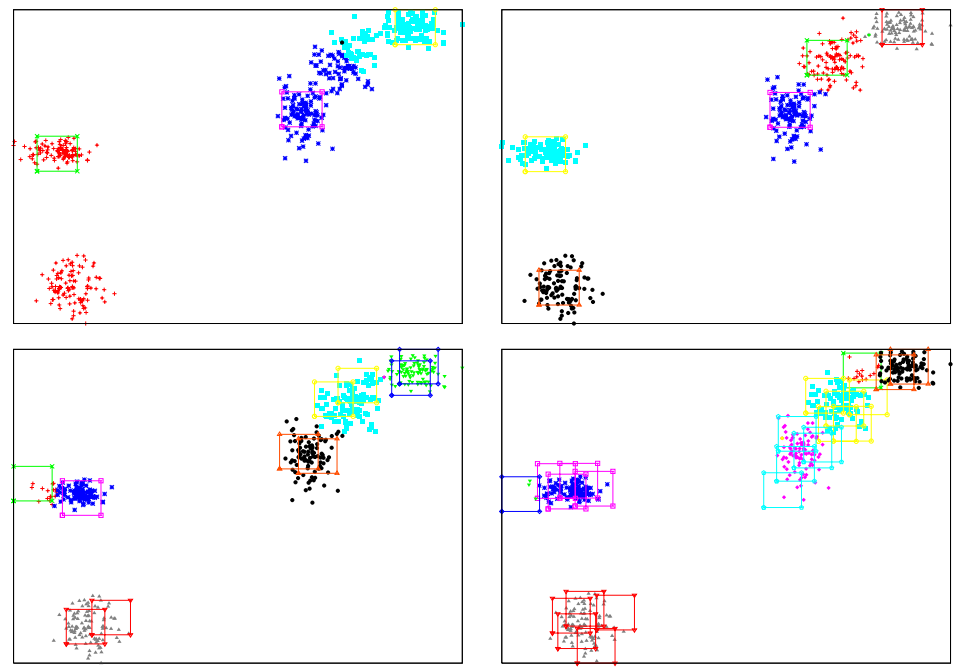

Fig. 20. The impact on the clustering result of different number of $d$-ranges $(3,5,10$ and 15) when $a=3$

Next, in Figure 20, we investigate the ability of DEUC to approximate the number of clusters. To this end we apply DEUC using 3, 5, 10 and 15 windows. As illustrated, when the number of $d$-ranges is less than the true number of clusters, each $d$-range is located over a minimum of WDF, but due to the inability to cover all the 


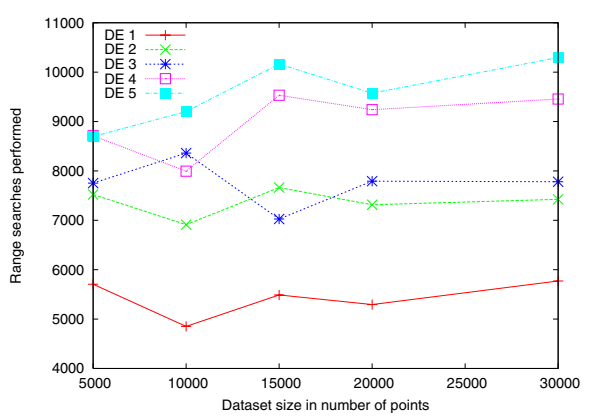

Fig. 21. Mean number of range searches required

minimizers the cluster labels are incorrect. On the other hand, as the number of $d$-ranges becomes larger than the real number of clusters, the algorithm has no problem of detecting the five clusters, since the merging procedure assigns correctly the cluster labels.

The complexity of the DEUC algorithm can be analyzed by the number of function evaluations it requires to provide a clustering result. As already mentioned for each function evaluation a range search operation over the dataset is performed. Measuring the total number of range searches that are needed is an indication of the relative speed of DEUC. To this end, we constructed Dset $_{2}$ in a manner similar to Dset $_{1}$, but with a size ranging from 5000 to 30000 points. The mean number of range searches required over 100 executions of DEUC, for all mutation operators is depicted in Figure 21

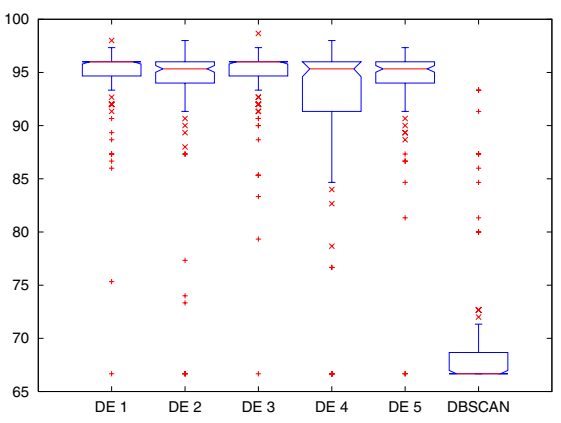

Fig. 22. Classification accuracy for the Iris dataset

From this figure it is clear that all the DE operators require a steady number of range searches to converge, irrespective of the dataset size. When the dataset size is small (5000) the number of ranges searches is relatively high. It even exceeds the total number of points. DBSCAN for each dataset requires at least $n$ range searches, where $n$ is the number of points in the dataset. It is evident that for small datasets DEUC appears computationally expensive. On the other hand, as the dataset size increases, the efficiency of DEUC also increases. For example for 30000 points in the dataset $\mathrm{DE}_{1}$ requires less than 6000 range searches, that is five times less than DBSCAN.

To demonstrate the quality of the partitioning results we employ the four dimensional Iris dataset $D$ set $_{\text {iris }}$ from the UCI Machine Learning Repository [10]. This dataset is among the best known databases to be found in the pattern recognition literature. It contains 150 records of four features. The features are measurements of the sepal and petal length and width of three different types of the iris plant (Setosa, Versicolour and Virginica). The 150 records are equally distributed in three classes, each corresponding to a different type of the plant. To evaluate the clustering result we resolve to the correspondence they have to the true cluster labels of the patterns. 
Ideally, each cluster should contain patterns that belong to only one type of the Iris plant. After normalizing the data in the $[10,100]^{4}$ range, DEUC was executed 100 times, using a population of 20 individuals while each individual encoded $5 d$-ranges. In most cases 3 clusters were recognized by the algorithm, but there were also cases that resulted in 4 and 5 clusters. Moreover, as a comparison measure we executed DBSCAN using all the combinations of values in $[1,10]$ with a step of 1, for the Eps and MinPts parameters, yielding 100 different clustering results. In the box-plots exhibited in Figure 22 we summarize the results with respect to the partitioning accuracy. As it is obvious from Figure 22 all the different DE operators are able to capture the dynamics of the dataset and result in high partitioning accuracy. Among all the operators $\mathrm{DE}_{3}$ exhibits the most robust behavior and is able to provide the best results even with respect to outliers. On the other hand, DBSCAN is unable to provide highly accurate results since in this dataset two of the classes are somewhat close and DBSCAN tends to merge them to a single cluster, thus destroying its classification accuracy.

In conclusion, it seems that it is possible using clustering criteria as the WDF density function, to design efficient and effective evolutionary clustering techniques. This is achieved by utilizing Computational Geometry data structures. Moreover, such an evolutionary procedure has the ability to approximate the number of clusters and yield high quality partitions as it is evident from the experimental results.

\section{Real Life Application: DNA Microarrays}

To understand a biological processes that a living cell undergoes, one has to measure the gene expression levels in different developmental phases, different body tissues, and different clinical conditions. Compared to the traditional approach to genomic research, which has been to examine and collect data for a single gene locally, DNA microarray technologies have rendered possible the simultaneous monitoring of the expression pattern of thousands of genes. Unfortunately, the original gene expression data are contaminated with noise, missing values and systematic variations due to the experimental procedure. Several methodologies can be employed to alleviate these problems, such as Singular Value Decomposition based methods, weighted $k$-nearest neighbors, row averages, replication of the experiments to model the noise, and/or normalization, which is the process of identifying and removing systematic sources of variation. Discovering the patterns hidden in the gene expression microarray data and subsequently using them to classify the various conditions is a tremendous opportunity and a challenge for functional genomics and proteomics. A promising approach to address this task is to utilize computational intelligence techniques, such as EAs and Feedforward Neural Networks (FNNs). Unfortunately, employing FNNs (or any other classifier) directly to classify the samples is almost infeasible due to the curse of dimensionality (limited number of samples coupled with very high feature dimensionality). One solution is to preprocess the expression matrix using a dimension reduction technique.

Here, we follow a different approach. DE and FNNs are employed to discover subsets of informative genes that accurately characterize all the samples [49]. Generally, the aim is to reduce the initial gene pool from several thousand genes $(5,000-10,000$ or more) to 50-100. Several gene selection methods based on statistical analysis have 
been developed to select these predictive genes and perform dimension reduction. Those methods include $t$-statistics, information gain theory, and principal component analysis (PCA). It is evident that the choice of feature selection is difficult and bears a significant effect on the overall classification accuracy. Typically, accuracy on the training data can be quite high, but not replicated on the testing data.

\subsection{Algorithms and Methodology}

To classify samples using microarray data, it is necessary to decide which genes, from the ones assayed, should be included in the classifier. Including too few genes and the test data will be incorrectly classified. On the other hand, having too many genes is not desirable either, as many of the genes will be irrelevant, mostly adding noise. This is particularly severe with a noisy data set and few subjects, as is the case with microarray data.

In the literature, both supervised and unsupervised classifiers have been used to build classification models from microarray data. This study addresses the supervised classification task where data samples belong to a known class. EAs are applied to microarray classification to determine the optimal, or near optimal, subset of predictive genes on complex and large spaces of possible gene sets. Although a vast number of gene subsets are evaluated by the EA, selecting the most informative genes is a non trivial task. Common problems include the existence of:

(a) relevant genes that are not included in the final subset, because of the insufficient exploration of the gene pool,

(b) significantly different subsets of genes being the most informative as the evolution progresses, and

(c) many subsets that perform equally well, as they all predict the test data satisfactorily.

From a practical point of view, the lack of a unique solution does not seem to present a problem.

The EA approach we describe maintains a population of trial gene subsets; imposes random changes on the genes that compose those subsets; and incorporates selection (driven by a neural network classifier) to determine which are the most informative ones. Only those genes are maintained in successive generations; the rest are removed from the trial pool. At each iteration, every subset is given as input to an FNN classifier and the effectiveness of the FNN determines the fitness of the subset of genes. The size of the population and the number of features in each subset are parameters that we explore experimentally.

For the outlined system, each population member represents a subset of genes, so a special representation must be designed. When seeking subsets containing $n$ genes, each individual consists of $n$ integers. The first integer is the index of the first gene to be included in the subset, the second integer denotes the number of genes to skip until the second gene to be included is reached, the third integer component denotes the number of genes to skip until the third included gene, and so on. This representation was necessary in order to avoid multiple inclusion of the same gene. Moreover, a version of DE that uses integer vectors has been thoroughly studied in previous Section. 
FNNs were used as a classifier to evaluate the fitness of each gene subset. One third of the data set is used as a training set for the FNN and one third is used to measure the classification accuracy of the FNN classifier. The remaining patterns of the data set are kept to estimate the classification capability of the final gene subset. All the FNNs were trained using the well known and widely used Resilient backpropagation (Rprop) [40] training algorithm. Rprop is a fast local adaptive learning scheme, performing supervised training. To update each weight of the FNN, Rprop exploits information concerning the sign of the partial derivative of the error function. In our experiments, the five parameters of the Rprop method were initialized using values commonly employed in the literature. In particular, the increase factor was set to $\eta^{+}=1.2$; the decrease factor was set to $\eta^{-}=0.5$; the initial update value is set to $\Delta_{0}=0.1$; the maximum step, which prevents the weights from becoming too large, was $\Delta_{\max }=50$; and the minimum step, which is used to avoid too small weight updates, was constantly fixed to $\Delta_{\min }=10^{-6}$.

\subsection{Presentation of Experiments in Evolutionary Dimension Reduction}

Next, we report the experimental results. We have tested and compared the performance of the described system on many publicly available microarray data sets. Here we report results from the following two data sets:

(a) The COLON data set [4] consists of 40 tumor and 22 normal colon tissues. For each sample there exist 2000 gene expression level measurements. The data set is available at http : / / microarray . princeton.edu / oncology

(b) The PROSTATE data set [13] contains 52 prostate tumor samples and 50 nontumor prostate samples. For each sample there exist 6033 gene expression level measurements. It is available at http : / / www . broad.mit . edu/cgi-bin/cancer/ dataset.cgi

Since the appropriate size of the most predictive gene set is unknown, DE was employed for various gene set sizes ranging from 10 to 100 with a step of 10 . The FNN used at the fitness function consisted of 2 hidden layers with eight and seven neurons, respectively. The input layer contained as many neurons as the size of the gene set. One output neuron was used at the output layer whose value for each sample determined the network classification decision. Since both problems had two different classes for the patterns, a value lower than 0.5 regarded the pattern to belong to class 1 otherwise regarded it to belong to class 2 .

For each different gene set size the data was partitioned randomly into a learning set consisting of two-thirds of the whole set and a test set consisting of the remaining one third, as already mentioned. The one third of the training set was used by the Rprop algorithm to train the FNNs, and the performance of the respective gene set was measured in the other one third. The test set was only used to evaluate the classification accuracy that can be obtained using the final gene set discovered by the DE algorithm. To reduce the variability, the splitting was repeated 10 times and 10 independent runs were performed each time, resulting in a total of 100 experiments, for gene set size.

The classification accuracy of the system is illustrated using boxplots in Figure 23 Each boxplot depicts the obtained values for the classification accuracy, in the 100 

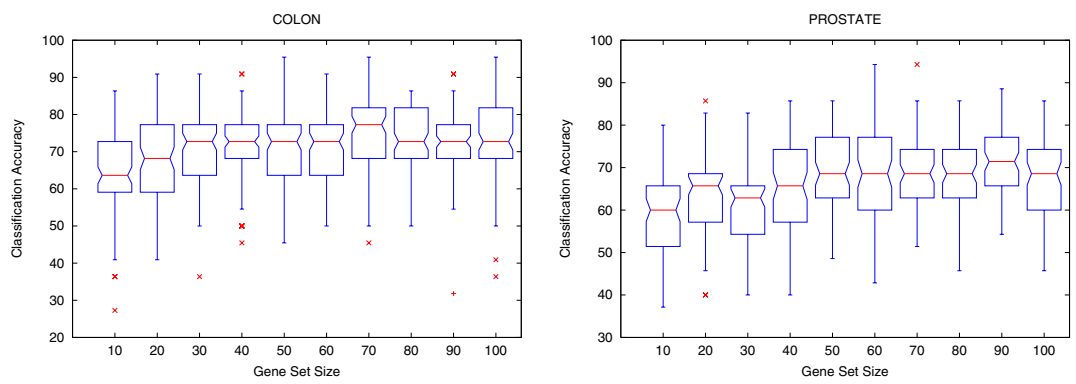

Fig. 23. Classification accuracy obtained by FNNs trained using the DE selected gene set for the COLON (left) and PROSTATE (right) datasets

experiments. As demonstrated, using a gene set size of 50-80 for the COLON dataset the algorithm managed to achieve the best results; comparable to those obtained by other approaches. The same is achieved for the PROSTATE dataset for a gene set size ranging from 40 to 60 .

\section{Future Directions: What Lies Ahead?}

More than ten years have passed since the initial proposal of DE by Storn and Price, and DE has been accepted as a strong the robust global optimization algorithm capable to handle nondifferentiable, nonlinear and multimodal objective functions. Although the driving force of $\mathrm{DE}$ are the mutation operators, little progress has been made to the extension of the algorithm by introducing new operators. Here, we utilize Genetic Programming (GP) to evolve novel DE mutation operators.

GP is a method for automatically creating working computer programs employing principles of Darwinian evolution, and having as input a high-level statement of the problem [28]. GP aspires to induce a population of computer programs that gradually improve as they evolve and experience the data on which they are evaluated. In this Section, we present a comparison between already known human-designed mutation operators and new genetically programmed ones. Our experimental results indicate that the performance of the genetically programmed operators is comparable and in some cases is considerably better than the already existing human designed ones. A genetically evolved operator also exhibited the most robust performance. Additionally, the genetic evolution resulted in parameter free mutation operators.

\subsection{Genetic Programming}

GP is an extension of Genetic Algorithms in which individuals are no longer fixedlength strings but rather computer programs expressed as syntax trees. GP individuals consist of function and terminal nodes. Terminal nodes store a value which they return as an output, while functions process their inputs to compute an output. The terminal set, $T$, is comprised of the inputs, the constants supplied, and the zero-argument functions. 
Thus, terminal nodes have an arity of zero. On the other hand, the function set, $F$, is composed of the statements and functions available to GP.

The primary GP search operators are crossover and mutation. In crossover, a randomly selected subtree from each of the two selected parents is exchanged between them to form two new individuals (offsprings). The idea is that useful building blocks for the solution of a problem are accumulated in the population and crossover permits the aggregation of good building blocks into even better solutions to the problem [29]. Crossover is the predominant search operator in GP [56]. Mutation operates on a single individual by altering a random subtree. Next, we briefly describe the GP initialization and the GP operators used.

\section{The GP Initialization}

The individuals in the GP population are initialized by recursively generating syntax trees composed of random function and terminal nodes. Two established GP initialization methods are the grow and the full method. Both methods require from the user to specify the maximum initial tree depth. According to the grow method, nodes are selected randomly from the function and the terminal sets. The grow method, therefore, produces trees of irregular shape, since once a terminal node is inserted the path ending with this node cannot be extended, even if the maximum initial depth has not been reached. On the other hand, in the full initialization method only function nodes are selected until the maximum initial depth is reached. Beyond that depth only terminal nodes are chosen to end the branches. This method results in a balanced tree, every branch of which reaches the maximum initial depth.

\section{The GP Selection Algorithm}

To derive the individuals that will comprise the population of the next generation, GP initially selects individuals from the current generation. The selection operators that have been proposed for Genetic Algorithms are also applicable to GP. In this study, we employed the most commonly encountered one, namely roulette wheel selection. Define the fitness of the $i$ th individual as $E_{i}$, where $E$ is the error function we wish to minimize. Then the probability of selecting individual $i$ as a parent of an individual of the next generation is equal to $E_{i}^{n} / \sum_{j=1}^{N} E_{j}^{n}$; where $E_{i}^{n}=1 /\left(1+E_{i}\right)$.

\section{The GP Crossover Operator}

The crossover operator combines the genetic material of two parents chosen by the selection operator to yield two offsprings. In particular, a real number $r$ is randomly chosen in the interval [0.1]. Crossover takes place only if $r \leqslant C$, where $C$ is the predefined crossover constant. In this case, a random node in each parent is chosen and the subtrees rooted at these nodes are exchanged between the parents to yield the offsprings. If an offspring exceeds the maximum depth it is discarded and the corresponding parent individual takes its place in the population of the next generation. Thus, crossover produces offsprings by swapping a part of one parent with a part of the other. If crossover does not take place $(r>C)$ the offsprings are exact copies of the parents. 


\section{The GP Mutation Operator}

After the crossover operator has finished, each offspring produced undergoes mutation. The probability of mutation is a user defined parameter. The mutation operator in GP randomly selects a node of the tree. If the node is a function then it is replaced by another function. If the node is terminal, another randomly selected terminal is used instead (point mutation) [56]. The mutated individual is then placed back into the population.

\subsection{Genetically Programmed Differential Evolution Mutation Operators}

As previously mentioned, in this section we investigate the discover of new efficient DE mutation operators using GP [33]. This is possible since, mutation operators are simply the composition of elementary functions such as addition, subtraction, and multiplication, operating on the vectors that represent individuals of the DE population. To this end, the terminal set used for GP, included two numerical constants, the vector of the best so far DE individual, $x_{g}^{\text {best }}$, three vectors of different randomly selected DE individuals, and the fixed mutation constant $\mu$ employed by the DE mutation operators. In detail, the terminal set used in this study was, $T=\left\{0.5,1, \mu, x_{g}^{\text {best }}, x_{g}^{r 1}, x_{g}^{r 2}, x_{g}^{r 3}\right\}$. The function set was $F=\{+,-, \odot, \oslash\}$, where $\odot$ and $\oslash$ are defined as follows:

$$
\begin{aligned}
& x \odot y=x^{\top} \cdot \operatorname{diag}\left\{y_{1}, y_{2}, \ldots, y_{n}\right\}=\left(x_{1} y_{1}, x_{2} y_{2}, \ldots, x_{n} y_{n}\right)^{\top} \\
& x \oslash y=x^{\top} \cdot \operatorname{diag}\left\{1 / y_{1}, 1 / y_{2}, \ldots, 1 / y_{n}\right\}=\left(x_{1} / y_{1}, x_{2} / y_{2}, \ldots, x_{n} / y_{n}\right)^{\top},
\end{aligned}
$$

where the vectors $x, y \in \mathbb{R}^{n}$, with $x^{\top}=\left(x_{1}, x_{2}, \ldots, x_{n}\right)$ and $y^{\top}=\left(y_{1}, y_{2}, \ldots, y_{n}\right)$. Note that the operator $\oslash$ utilizes a protected division; if the absolute value of the denominator is less 0.0001 , then $\oslash$ returns 1 .

The presentation of the problem and the fitness function typically define the space of candidate solutions for each particular problem. At present, more than one performance measure are applicable. One approach is to use the distance of the discovered minimizer from the global one to measure the operator's performance [36]. However, in many real life applications the location of the global minimizer is unknown. Conversely, the value of the global minimum could be known (for example when minimizing the sum of squares, a chemical or physical process, etc.).

In this study, we defined a fitness function, suitable for general optimization tasks, which utilizes three benchmark optimization problems discussed in previous Section: the Shekel's Foxholes, the Corana Parabola and the Levy No. 5 test problems. More specifically, the performance of each operator was measured through the sum of the generations required to locate the global minimum on each benchmark function, plus the minimum function values that were discovered. It is known that the performance of the DE algorithm (like the performance of every other EA) can vary with the initial random individuals. To reduce the effect of the stochastic nature of DE, 10 independent evaluations were performed, and the final fitness was averaged. If the global minimum was not found after 100 generations DE terminated. Using this fitness function, we strain GP evolution towards obtaining DE operators capable of locating the global optimum, within a minimum number of generations. 


\subsection{Experimental Discovery of Genetically Programmed Operators}

The computational experiments were performed utilizing a novel GP-DE interface. We employed the full GP initialization method with a maximum initial tree depth of 3. Another critical GP parameter is the maximum allowed depth for the trees. The maximum depth parameter is the largest allowed depth between the root node and the outermost terminals. The maximum depth during the GP execution was 100. GP population size was 40 , while the maximum number of generations was set to 1000 . The mutation and crossover probabilities for GP were set to 0.6 and 0.1 , respectively. The values for the parameters $\mu$ and $\rho$ employed by the DE algorithm (irrespective of the mutation operator), were set to 0.6 and 0.8 , respectively.

We conducted 100 independent GP experiments. The five best performing DE mutation operators discovered are the following:

$$
\begin{aligned}
v_{g+1}^{i} & =\left(x_{g}^{r 1}+x_{g}^{r 2}\right) \oslash\left(1+\left(x_{g}^{r 3} \oslash x_{g}^{\text {best }}\right)\right), \\
v_{g+1}^{i} & =x_{g}^{\text {best }}+0.5\left(x_{g}^{r 1}-x_{g}^{r 2}\right), \\
v_{g+1}^{i} & =\left(x_{g}^{r 1}+x_{g}^{r 3}\right) \oslash\left(\left(x_{g}^{r 1} \oslash x_{g}^{\text {best }}\right)+\left(x_{g}^{r 2} \oslash x_{g}^{\text {best }}\right)\right), \\
v_{g+1}^{i} & =\left(x_{g}^{r 3}+x_{g}^{\text {best }}\right) \oslash\left(\left(x_{g}^{r 3} \oslash x_{g}^{r 1}\right)+\left(x_{g}^{r 2} \oslash x_{g}^{\text {best }}\right)\right), \\
v_{g+1}^{i} & =\left(\left(x_{g}^{r 1} \odot x_{g}^{r 3}\right) \oslash\left(x_{g}^{r 1}+x_{g}^{r 3}\right)\right) \odot\left(\left(x_{g}^{\text {best }} \oslash x_{g}^{r 3}\right)+\left(x_{g}^{\text {best }} \oslash x_{g}^{r 2}\right)\right) .
\end{aligned}
$$

Throughout the remaining chapter, we call $\mathrm{GPDE}_{1}, \mathrm{GPDE}_{2}, \ldots, \mathrm{GPDE}_{5}$ the DE algorithm that uses Equation (8), Equation (9), .., Equation (12) as the mutation operator, respectively. It is evident that this methodology allows us to routinely "invent" new specialized DE operators, which are optimal or near-optimal for a specific problem. Notice that although the mutation constant $\mu$ was included in the terminal set, all the above mentioned GP derived DE mutation operators are parameter free. This is a considerable advantage since it alleviates the need for parameter tuning by the user.

The original DE algorithm exploits the information from the differences between pairs of individuals to guide its search in the solution domain. Although, in all the mutation operators discovered here, individuals interact in pairs, pairwise differences are not encountered in any GPDE operator but $\mathrm{GPDE}_{2}$. Indeed, $\mathrm{GPDE}_{2}$ is equivalent to $\mathrm{DE}_{1}$ for the special case that $\mu=0.5$. The experimental results reported below suggest that this particular setting is more effective than a typical value of $\mu$ for the benchmark problems considered. Note that $\mathrm{DE}_{1}$ has been documented as one of the most effective and robust mutation operators. It is also interesting to note that the best individual of the current generation appears at least once in all GPDE operators.

To measure the efficiency and effectiveness of the newly discovered GPDE operators, we tested them on the three previously mentioned optimization benchmark functions, as well as on two additional functions; namely the Griewangk's and the Rosenbrock's Saddle test functions.

The performance of the human-designed and the genetically programmed DE mutation operators is presented in Table 7 In particular, for each mutation operator and for each benchmark function, Table 7 reports the mean number of generations required to locate a global minimizer (Gen.), as well as, the percentage of times the algorithm was 
Table 7. Human Designed vs. Genetically Programmed Differential Evolution Operators

\begin{tabular}{|c|c|c|c|c|c|c|c|c|c|c|}
\hline & \multicolumn{5}{|c|}{\begin{tabular}{|l} 
TRAINING PHASE \\
\end{tabular}} & EM 3 & PROI & $\begin{array}{l}\text { STIN } \\
\text { EM } 1\end{array}$ & $\begin{array}{l}\text { G PH } \\
\text { Pro }\end{array}$ & M 2 \\
\hline & Gen. & $(\%)$ & Gen. & $(\%)$ & Gen. & $(\%)$ & Gen. & $(\%)$ & Gen. & $(\%)$ \\
\hline $\mathrm{DE}_{1}$ & 95.6 & 5 & 87.5 & 42 & 64.9 & 50 & 29.8 & 100 & - & 0 \\
\hline $\mathrm{DE}_{2}$ & 83.8 & 95 & - & 0 & 76.8 & 100 & 56.8 & 100 & - & 0 \\
\hline $\mathrm{DE}_{3}$ & 97.5 & 13 & 97.9 & 35 & 76.9 & 63 & 43.7 & 100 & - & 0 \\
\hline $\mathrm{DE}_{4}$ & - & 0 & 39.8 & 79 & 89.2 & 16 & 52.3 & 74 & 96.8 & 12 \\
\hline $\mathrm{DE}_{5}$ & 94.6 & 20 & - & 0 & 92.9 & 22 & 81.2 & 85 & - & 0 \\
\hline $\mathrm{DE}_{6}$ & 81.2 & 64 & - & 0 & 72.1 & 100 & 63.1 & 96 & - & 0 \\
\hline $\mathrm{GPDE}_{1}$ & - & 0 & 29.9 & 94 & 43.7 & 96 & 55.9 & 68 & 93.6 & 44 \\
\hline $\mathrm{GPDE}_{2}$ & 84.1 & 23 & 98.8 & 3 & 34.0 & 88 & 25.1 & 100 & - & 0 \\
\hline $\mathrm{GPDE}_{3}$ & 78.5 & 25 & 58.2 & 57 & 52.8 & 65 & 28.0 & 100 & 76.9 & 53 \\
\hline $\mathrm{GPDE}_{4}$ & 一 & 0 & 12.1 & 100 & 82.6 & 32 & 38.8 & 100 & 20.3 & 100 \\
\hline $\mathrm{GPDE}_{5}$ & - & 0 & 25.7 & 100 & 57.1 & 59 & 38.4 & 93 & 82.2 & 28 \\
\hline
\end{tabular}

( - denotes that the algorithm failed to find the global minimum in all runs).

successful in locating a global minimizer $(\%)$. The reported results are averages over 100 independent experiments for each mutation operator. Note, that in the cases DE was unable to identify a global minimizer, the maximum allowed number of generations was added to the sum used to compute the mean number of generations required to locate a global minimizer. The entry "_-" in the table suggests that the success rate of a mutation operator for the corresponding benchmark was zero. Finally, bold faced entries are used to indicate the mutation operator with the lowest mean number of generations to detect a global minimizer and the one with the highest success rate.

With respect to the mean number of generations required to detect a global minimizer, the best performing mutation operator is in all cases derived by Genetic Programming. The best performing mutation strategy in this respect is $\mathrm{GPDE}_{2}$, which is a special case of $\mathrm{DE}_{1}$. For two out the five optimization problems (Train Problem 3 and Test Problem 1), $\mathrm{DE}_{1}$ requires the lowest mean number of generations to compute a global minimizer among the original DE operators. On the same two problems $\mathrm{GPDE}_{2}$ is the overall best performing strategy in this respect, but it performs badly on Test Problem 2. GPDE 4 is by far the best performing strategy on Train Problem 2 and Test Problem 2, for which most operators performed badly. With respect to the percentage of times a minimizer was located, the two types of operators perform similarly well on Test Problem 1. Last but not least, it is important to note that the most robust operator with respect to both criteria is $\mathrm{GPDE}_{3}$. It is the best performing operator with respect to mean number of generations on Train Problem 1, the second best on Test Problem 1, and the third best performing on Train Problem 3 and Test Problem 2. Furthermore, it is the only operator that achieved a positive percentage of locating a minimizer on all the test functions. Our experience is that $\mathrm{GPDE}_{3}$ is stable and effective, and can be used to optimize an unknown function with good results.

In accordance to the "no free lunch theorem" [57], it is impossible to find a single DE operator that outperforms all the other in every test problem. Instead, here we try to discover new DE operators better suited for general optimization problems, or classes 
of problems. The experimental results indicate that the best performing DE mutation operator is in all cases GP derived. GP has been able to automatically evolve a variety of new DE mutation operators that operate as well or considerably better, for the considered problems, than the already existing human-designed ones. It is interesting to note that all the new DE mutation operators are parameter free, in the sense that no mutation constant is needed.

\section{Synopsis}

In this chapter we presented an overview of the major applications areas of differential evolution. The DE algorithms have shown their strength in tackling many difficult problems from diverse scientific areas, including single and multiobjective function optimization, neural network training, clustering, and real life DNA microarray classification.

All the experiments presented in this chapter have been performed using distributed computing environments, since DE can be easily parallelized in a virtual parallel environment so as to improve both its speed and performance. The results indicate that the extent of information exchange among subpopulations assigned to different processor nodes, aids the algorithm to converge faster and find better solutions. To demonstrate that we have introduced the parallel, multi-population DE algorithm for single and multiobjective optimization.

Next, we presented a case where DE can be utilized to perform data clustering. Additionally, clustering algorithms can also aid DE to locate simultaneously multiple local and global minimizers of an objective function. This can be accomplished by the new clustering operator. This operator incorporates the unsupervised $k$-windows clustering algorithm, utilizing already computed pieces of information regarding the search space in an attempt to discover regions containing groups of individuals located close to different minimizers. Then, the search is confined inside these regions and a large number of global and local minimizers of the objective function can be efficiently computed.

The real life DE applications presented here include the training of integer weight neural networks with threshold activations and the selection of genes of DNA microarrays in order to obtain high predictive accuracy subsets. In both cases, the DE addressed problems of very high dimensionality successfully.

We closed this chapter with a discussion on promising future extensions of the algorithm by the incorporation of genetically programmed mutation operators. These operators improve the quality of the solutions and accelerate the execution of the algorithm. It must be noted that the genetic evolution resulted in parameter free DE operators. This is a considerable advantage since it alleviates the need for parameter tuning by the user. The results indicate that the performance of the genetically programmed operators is comparable and in some cases is considerably better than the already existing human designed ones. We feel that this can be a very interesting future research direction. 


\section{References}

1. MPI the message passing interface standard, http://www-unix.mcs .anl.gov/mpi/

2. Abbass, H.: Self-adaptive pareto differential evolution. In: Proceedings of the IEEE 2002 Congress on Evolutionary Computation, Honolulu, Hawaii, pp. 831-836. IEEE Press, Los Alamitos (2002)

3. Alevizos, P.: An algorithm for orthogonal range search in $d \geqslant 3$ dimensions. In: Proceedings of the 14th European Workshop on Computational Geometry. Barcelona (1998)

4. Alon, U., Barkai, N., Notterman, D.A., Gish, K., Ybarra, S., Mack, D., Levine, A.J.: Broad patterns of gene expression revealed by clustering analysis of tumor and normal colon tissues probed by oligonucleotide array. Proc. Natl. Acad. Sci. USA 96(12), 6745-6750 (1999)

5. Babu, G.P., Murty, M.N.: A near optimal initial seed value selection in $k$-means algorithm using a genetic algorithm. Pattern Recogn. Lett. 14(10), 763-769 (1993)

6. Babu, G.P., Murty, M.N.: Clustering with evolution strategies. Pattern Recogn. 27, 321-329 (1994)

7. Becker, R.W., Lago, G.V.: A global optimization algorithm. In: Proceedings of the 8th Allerton Conference on Circuits and Systems Theory, pp. 3-12 (1970)

8. Bentley, J.L., Maurer, H.A.: Efficient worst-case data structures for range searching. Acta Informatica 13, 1551-1568 (1980)

9. Bhuyan, J.N., Raghavan, V.V., Venkatesh, K.E.: Genetic algorithm for clustering with an ordered representation. In: Fourth International Conference on Genetic Algorithms, pp. 408 415 (1991)

10. Blake, C.L., Merz, C.J.: UCI repository of machine learning databases (1998)

11. Coello Coello, C.A., Van Veldhuizen, D.A., Lamont, G.B.: Evolutionary Algorithms for Solving Multi-Objective Problems. Kluwer, New York (2002)

12. Deb, K.: Multi-objective genetic algorithms: Problem difficulties and construction of test problems. Evolutionary Computation 7(3), 205-230 (1999)

13. Singh, D., et al.: Gene expression correlates of clinical prostate cancer behavior. Cancer Cell 1, 203-209 (2002)

14. Fan, H.Y., Lampinen, J.: A trigonometric mutation operation to differential evolution. Journal of Global Optimization 27, 105-129 (2003)

15. Fayyad, U.M., Piatetsky-Shapiro, G., Smyth, P.: Advances in Knowledge Discovery and Data Mining. MIT Press, Cambridge (1996)

16. Fieldsend, J.E., Everson, R.M., Singh, S.: Using unconstrained elite archives for multiobjective optimization. IEEE Trans. Evol. Comp. 7(3), 305-323 (2003)

17. Fogel, D.: Evolutionary Computation: Towards a New Philosophy of Machine Intelligence. IEEE Press, Piscataway (1996)

18. Fogel, D.B., Simpson, P.K.: Evolving fuzzy clusters. In: International Conference on Neural Networks, pp. 1829-1834 (1993)

19. Fogel, L.J., Owens, A.J., Walsh, M.J.: Artificial intelligence through simulated evolution. Wiley, Chichester (1966)

20. Geist, A., Beguelin, A., Dongarra, J., Jiang, W., Manchek, R., Sunderam, V.: PVM: Parallel Virtual Machine. A Users Guide and Tutorial for Networked Parallel Computing. MIT Press, Cambridge (1994)

21. Goldberg, D.: Genetic Algorithms in Search, Optimization, and Machine Learning. Addison Wesley, Reading (1989)

22. Handl, J., Knowles, J.: Evolutionary multiobjective clustering. In: Yao, X., Burke, E.K., Lozano, J.A., Smith, J., Merelo-Guervós, J.J., Bullinaria, J.A., Rowe, J.E., Tiňo, P., Kabán, A., Schwefel, H.-P. (eds.) PPSN 2004. LNCS, vol. 3242, pp. 1081-1091. Springer, Heidelberg (2004) 
23. Haykin, S.: Neural Networks. Macmillan College Publishing Company, New York (1999)

24. Holland, J.H.: Adaptation in natural and artificial system. University of Michigan Press (1975)

25. Jin, Y., Olhofer, M., Sendhoff, B.: Evolutionary dynamic weighted aggregation for multiobjective optimization: Why does it work and how? In: Proceedings GECCO 2001 Conference, San Francisco, CA, pp. 1042-1049 (2001)

26. Jones, D., Beltramo, M.A.: Solving partitioning problems with genetic algorithms. In: Fourth International Conference on Genetic Algorithms, pp. 442-449 (1991)

27. Kennedy, J., Eberhart, R.C.: Particle swarm optimization. In: Proceedings IEEE International Conference on Neural Networks, Piscataway, NJ, vol. IV, pp. 1942-1948. IEEE Service Center (1995)

28. Koza, J.R.: Hierarchical genetic algorithms operating on populations of computer programs. In: Proceedings of the Eleventh International Joint Conference on Artificial Intelligence, pp. 768-774 (1989)

29. Koza, J.R.: Genetic Programming: On the Programming of Computers by Means of Natural Selection. MIT Press, Cambridge (1992)

30. Laumanns, M., Zitzler, E., Thiele, L.: A unified model for multiobjective evolutionary algorithms with elitism. In: Proc. IEEE Congr. Evol. Comp., Piscataway, NJ, pp. 46-53. IEEE Press, Los Alamitos (2000)

31. Marriott, F.H.C.: Optimisation methods of cluster analysis. Biometrics 69(2), 417-422 (1982)

32. Michalewicz, Z., Fogel, D.B.: How to solve it: Modern Heuristics. Springer, Heidelberg (2000)

33. Pavlidis, N.G., Tasoulis, D.K., Plagianakos, V.P., Vrahatis, M.N.: Human designed vs. genetically programmed differential evolution operators. In: IEEE Congress on Evolutionary Computation, pp. 1880-1886 (2006)

34. Plagianakos, V.P., Vrahatis, M.N.: Training neural networks with threshold activation functions and constrained integer weights. In: IEEE International Joint Conference on Neural Networks (IJCNN 2000), Como, Italy (2000)

35. Plagianakos, V.P., Vrahatis, M.N.: Parallel evolutionary training algorithms for 'hardwarefriendly' neural networks. Natural Computing 1, 307-322 (2002)

36. Poli, R., Langdon, W.B., Holland, O.: Extending particle swarm optimisation via genetic programming. In: Keijzer, M., Tettamanzi, A.G.B., Collet, P., van Hemert, J.I., Tomassini, M. (eds.) EuroGP 2005. LNCS, vol. 3447. Springer, Heidelberg (2005)

37. Preparata, F., Shamos, M.: Computational Geometry. Springer, New York (1985)

38. Ramasubramanian, V., Paliwal, K.: Fast $k$-dimensional tree algorithms for nearest neighbor search with application to vector quantization encoding. IEEE Transactions on Signal Processing 40(3), 518-531 (1992)

39. Rechenberg, I.: Evolution strategy. In: Zurada, J.M., Marks II, R.J., Robinson, C. (eds.) Computational Intelligence: Imitating Life. IEEE Press, Piscataway (1994)

40. Riedmiller, M., Braun, H.: A direct adaptive method for faster backpropagation learning: The rprop algorithm. In: Proceedings of the IEEE International Conference on Neural Networks, San Francisco, CA, pp. 586-591 (1993)

41. Sander, J., Ester, M., Kriegel, H.-P., Xu, X.: Density-based clustering in spatial databases: The algorithm gdbscan and its applications. Data Mining and Knowledge Discovery 2(2), 169-194 (1998)

42. Schaffer, J.D.: Multiple Objective Optimization With Vector Evaluated Genetic Algorithms. PhD thesis, Vanderbilt University, Nashville, TN, USA (1984)

43. Schwefel, H.-P.: Evolution and Optimum Seeking. Wiley, New York (1995)

44. Steinbach, M., Karypis, G., Kumar, V.: A comparison of document clustering techniques. In: KDD Workshop on Text Mining (2000) 
45. Storn, R.: System design by constraint adaptation and differential evolution. IEEE Transactions on Evolutionary Computation 3, 22-34 (1999)

46. Storn, R., Price, K.: Differential evolution - a simple and efficient adaptive scheme for global optimization over continuous spaces. Journal of Global Optimization 11, 341-359 (1997)

47. Tasoulis, D.K., Pavlidis, N.G., Plagianakos, V.P., Vrahatis, M.N.: Parallel differential evolution. In: IEEE Congress on Evolutionary Computation (CEC 2004) (2004)

48. Tasoulis, D.K., Plagianakos, V.P., Vrahatis, M.N.: Clustering in evolutionary algorithms to efficiently compute simultaneously local and global minima. In: IEEE Congress on Evolutionary Computation, pp. 1847-1854 (2005)

49. Tasoulis, D.K., Plagianakos, V.P., Vrahatis, M.N.: Differential evolution algorithms for finding predictive gene subsets in microarray data. In: Artificial Intelligence Applications and Innovations. IFIP International Federation for Information Processing, vol. 204, pp. 484491 (2006)

50. Tasoulis, D.K., Vrahatis, M.N.: Novel approaches to unsupervised clustering through the $k$ windows algorithm. In: Sirmakessis, S. (ed.) Knowledge Mining. Studies in Fuzziness and Soft Computing, vol. 185, pp. 51-78. Springer, Heidelberg (2005)

51. Tasoulis, D.K., Vrahatis, M.N.: Unsupervised clustering on dynamic databases. Pattern Recognition Letters 26(13), 2116-2127 (2005)

52. Torn, A., Zilinskas, A.: Global Optimization. Springer, Berlin (1989)

53. van der Merwe, D.W., Engelbrecht, A.P.: Data clustering using particle swarm optimization. In: Congress on Evolutionary Computation, Canberra, Australia, pp. 215-220 (2003)

54. Van Veldhuizen, D.A., Zydallis, J.B., Lamont, G.B.: Considerations in engineering parallel multiobjective evolutionary algorithms. IEEE Trans. Evol. Comp. 7(2), 144-173 (2003)

55. Vrahatis, M.N., Boutsinas, B., Alevizos, P., Pavlides, G.: The new $k$-windows algorithm for improving the $k$-means clustering algorithm. Journal of Complexity 18, 375-391 (2002)

56. Wolfgang, B., Nordin, P., Keller, R.E., Francone, F.D.: Genetic programming: An Introduction: on the automatic evolution of computer programs and its applications. Morgan Kaufmann Publishers Inc., San Francisco (1998)

57. Wolpert, D.H., Macready, W.G.: No free lunch theorems for optimization. IEEE Transactions on Evolutionary Computation 1(1), 67-82 (1997)

58. Yang, M.-S., Wu, K.-L.: A similarity-based robust clustering method. IEEE Transactions on Pattern Analysis and Machine Intelligence 26(4), 434-448 (2004)

59. Zitzler, E.: Evolutionary Algorithms for Multiobjective Optimization: Methods and Applications. PhD thesis, Swiss Federal Institute of Technology Zürich, Switzerland (1999)

60. Zitzler, E., Deb, K., Thiele, L.: Comparison of multiobjective evolution algorithms: Empirical results. Evolutionary Computation 8(2), 173-195 (2000) 